\title{
Catalytic Asymmetric Vinylation of Ketones
}

\author{
Hongmei Li and Patrick J. Walsh* \\ P. Roy and Diane T. Vagelos Laboratories, \\ University of Pennsylvania, Department of Chemistry \\ 231 South 34th Street, Philadelphia, PA 19104-6323.
}

\section{Supporting Information}

Table of Contents $\quad$ Page

$\begin{array}{ll}\text { General Methods } & \text { S2 }\end{array}$

General Procedure for the Preparation of Allylic Alcohols $\quad$ S2

$\begin{array}{ll}\text { Characterization of } \mathbf{S 1} \mathbf{-} \mathbf{S 1 5} & \text { S2 }\end{array}$

Conditions for the Determination of Enantiomeric Excess $\quad$ S13

$\begin{array}{ll}\text { Reference } & \text { S15 }\end{array}$

${ }^{1} \mathrm{H}$ and ${ }^{13} \mathrm{C}\left\{{ }^{1} \mathrm{H}\right\}$ NMR spectra $\quad \mathrm{S} 16$ 
General Methods. All reactions were carried out under a nitrogen atmosphere using standard Schlenk techniques. All chemicals were obtained from Aldrich or Acros Organics unless otherwise specified. All solvents were purchased from Fischer Scientific. $\mathrm{Cp}_{2} \mathrm{ZrHCl}$ and $\mathrm{Me}_{2} \mathrm{Zn} 2.0 \mathrm{M}$ toluene solution were purchased from the Aldrich Chemical Company. Titanium (IV) isopropoxide and all liquid ketone substrates were distilled prior to use. Titanium (IV) isopropoxide (1.4 $\mathrm{M}$ in toluene) was prepared and stored in a Vacuum Atmospheres dry box. Toluene, dichloromethane, and hexanes were dried through activated alumina columns. NMR spectra were obtained on a Brüker $500 \mathrm{MHz}$ Fourier transform spectrometer at the University of Pennsylvania NMR facility. ${ }^{1} \mathrm{H}$ NMR spectra were referenced to tetramethylsilane in $\mathrm{CDCl}_{3} ;{ }^{13} \mathrm{C}\left\{{ }^{1} \mathrm{H}\right\}$ NMR spectra were referenced to residual solvent. The infrared spectra were obtained using a Perkin-Elmer 1600 series spectrometer.

General Protocol A. Preparation of 2-phenyl-oct-3-en-2-ol (S1).

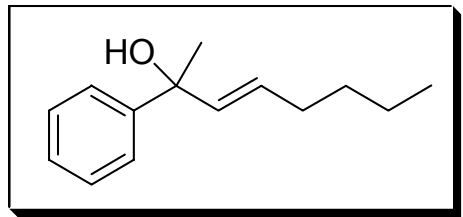

To a suspension of $\mathrm{Cp}_{2} \mathrm{ZrHCl}(155 \mathrm{mg}, 0.60 \mathrm{mmol})$ in $\mathrm{CH}_{2} \mathrm{Cl}_{2}(2.0 \mathrm{~mL})$ under $\mathrm{N}_{2}$ was added 1-hexyne $(70 \mu \mathrm{L}$, $0.60 \mathrm{mmol}$ ) and the reaction mixture was stirred for $10 \mathrm{~min}$ at r.t. after which it was a homogeneous yellow solution. The solvent was removed in vacuo and the residue was dissolved in dry toluene $(2.0 \mathrm{~mL})$, cooled to $-78^{\circ} \mathrm{C}$, treated with $\mathrm{Me}_{2} \mathrm{Zn}(0.30 \mathrm{~mL}, 2.0 \mathrm{M}$ in toluene, $0.60 \mathrm{mmol})$ for $10 \mathrm{~min}$. In another Schlenk flask were mixed ligand $1(27.3 \mathrm{mg}, 0.05 \mathrm{mmol}, 10 \mathrm{~mol} \%)$ in $1.0 \mathrm{~mL}$ toluene, $\mathrm{Me}_{2} \mathrm{Zn}(0.10$ $\mathrm{mL}, 2.0 \mathrm{M}$ in toluene, $0.20 \mathrm{mmol})$ and $\operatorname{Ti}\left(\mathrm{O}^{i} \operatorname{Pr}\right)_{4}(0.43 \mathrm{~mL}, 1.4 \mathrm{M}$ toluene solution, 0.60 mmol) at r.t. and stirred for 15 min. The resulting solution was added to the Schlenk flask 
containing the vinylzirconocene and dimethylzinc at $-78^{\circ} \mathrm{C}$. After the addition, the solution was warmed to $0^{\circ} \mathrm{C}$, and acetophenone $(59 \mu \mathrm{L}, 0.50 \mathrm{mmol})$ was added. The reaction mixture was warmed to r.t. and stirred until TLC showed complete consumption of the ketone. The reaction mixture was quenched with saturated $\mathrm{NaHCO}_{3}(5 \mathrm{~mL})$, diluted with EtOAc, filtered through Celite, and the layers were separated. The aqueous layer was extracted with EtOAc $(2 \times 40 \mathrm{~mL})$ and the combined organic layers were washed with brine, dried over $\mathrm{MgSO}_{4}$, and concentrated. The residue was purified by flash chromatography on deactivated silica gel $\left(\mathrm{Et}_{3} \mathrm{~N} / \mathrm{SiO}_{2}=2.5 \% \mathrm{~V} / \mathrm{V}\right.$, hexanes : EtOAc / 96:4) to give $\mathbf{S 1}\left(103 \mathrm{mg}, 85 \%\right.$ yield, $93 \%$ ee) as an oil: $[\alpha]_{\mathrm{D}}{ }^{20}=+0.4\left(c 2.0, \mathrm{CHCl}_{3}\right) ;{ }^{1} \mathrm{H}$ $\operatorname{NMR}\left(\mathrm{CDCl}_{3}, 500 \mathrm{MHz}\right) \delta 0.89(\mathrm{t}, J=7.1 \mathrm{~Hz}, 3 \mathrm{H}), 1.26-1.40(\mathrm{~m}, 4 \mathrm{H}), 1.62(\mathrm{~s}, 3 \mathrm{H})$, 2.03-2.09 (m, 2H), 5.63-5.69 (m, 1H), $5.77(\mathrm{~d}, J=15.5 \mathrm{~Hz}, 1 \mathrm{H}), 7.21-7.24(\mathrm{~m}, 1 \mathrm{H}), 7.32$ $(\mathrm{dd}, J=7.9,7.5 \mathrm{~Hz}, 2 \mathrm{H}), 7.45(\mathrm{~d}, J=7.4 \mathrm{~Hz}, 2 \mathrm{H}) \mathrm{ppm} ;{ }^{13} \mathrm{C}\left\{{ }^{1} \mathrm{H}\right\} \mathrm{NMR}\left(\mathrm{CDCl}_{3}, 125 \mathrm{MHz}\right)$ $\delta 14.4,22.7,30.4,31.8,32.3,74.8,125.6,127.2,128.5,129.5,137.3,147.8$ ppm; IR (film) 3388, 3025, 2956, 2874, 2358, 1667, 1601, 1492, 1446, $1369 \mathrm{~cm}^{-1}$; HRMS-CI m/z $187.1477\left[(\mathrm{M}-\mathrm{OH})^{+}\right.$; calcd for $\left.\mathrm{C}_{14} \mathrm{H}_{19}: 187.1487\right]$; HRMS-CI $m / z 186.1403\left[\left(\mathrm{M}-\mathrm{H}_{2} \mathrm{O}\right)^{+}\right.$; calcd for $\left.\mathrm{C}_{14} \mathrm{H}_{18}: 186.1408\right]$.

\section{Preparation of 4-cyclopropyl-2-phenyl-but-3-en-2-ol (S2).}

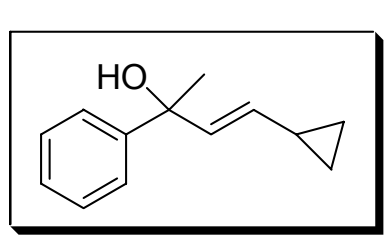

The product was prepared by General Procedure A using $\mathrm{Cp}_{2} \mathrm{ZrHCl}$ (155 mg, $\left.0.60 \mathrm{mmol}\right)$, cyclopropyl acetylene (40 mg, $0.60 \mathrm{mmol}), \mathrm{Me}_{2} \mathrm{Zn}(0.30 \mathrm{~mL}, 0.60 \mathrm{mmol}, 2.0 \mathrm{M}$ in toluene), ligand 1 (27.3 mg, $0.05 \mathrm{mmol}, 10 \mathrm{~mol} \%), \mathrm{Me}_{2} \mathrm{Zn}(0.10 \mathrm{~mL}, 0.20 \mathrm{mmol}, 2.0 \mathrm{M}$ in toluene), $\mathrm{Ti}\left(\mathrm{O}^{i} \mathrm{Pr}\right)_{4}(0.43 \mathrm{~mL}, 0.60 \mathrm{mmol}, 1.4 \mathrm{M}$ toluene solution) and acetophenone (59 
$\mu \mathrm{L}, 0.50 \mathrm{mmol})$. The crude product was purified by column chromatography on deactivated silica gel $\left(\mathrm{Et}_{3} \mathrm{~N} / \mathrm{SiO}_{2}=2.5 \% \mathrm{~V} / \mathrm{V}\right.$, hexanes : EtOAc / 95:5) to give $\mathbf{S 2}(85 \mathrm{mg}$, $90 \%$ yield, $95 \%$ ee $)$ as an oil: $[\alpha]_{\mathrm{D}}{ }^{20}=-5.0\left(\right.$ c 2.00, $\left.\mathrm{CHCl}_{3}\right) ;{ }^{1} \mathrm{H} \mathrm{NMR}\left(\mathrm{CDCl}_{3}, 500 \mathrm{MHz}\right)$ $\delta$ 0.35-0.36 (m, 2H), 0.68-0.70 (m, 2H), 1.36-1.40 (m, 1H), $1.60(\mathrm{~s}, 3 \mathrm{H}), 5.14-5.19(\mathrm{~m}$, 1H), $5.84(\mathrm{~d}, J=15.5 \mathrm{~Hz}, 1 \mathrm{H}), 7.20-7.25(\mathrm{~m}, 1 \mathrm{H}), 7.29-7.32(\mathrm{~m}, 2 \mathrm{H}), 7.40-7.45(\mathrm{~m}, 2 \mathrm{H})$ ppm; ${ }^{13} \mathrm{C}\left\{{ }^{1} \mathrm{H}\right\} \mathrm{NMR}\left(\mathrm{CDCl}_{3}, 125 \mathrm{MHz}\right) \delta 7.2,14.0,30.4,74.7,125.7,127.2,128.5$, 133.4, 135.1, 147.8 ppm; IR (film) 3391, 3081, 3004, 2977, 2927, 1663, 1600, 1493, 1446, $1369 \mathrm{~cm}^{-1}$; HRMS-CI $m / z 188.1194$ [ $\mathrm{M}^{+}$; calcd for $\left.\mathrm{C}_{13} \mathrm{H}_{16} \mathrm{O}: 188.1201\right]$.

\section{Preparation of 5-(tert-butyl-diphenyl-silanyloxy)-2-phenyl-pent-3-en-2-ol (S3).}

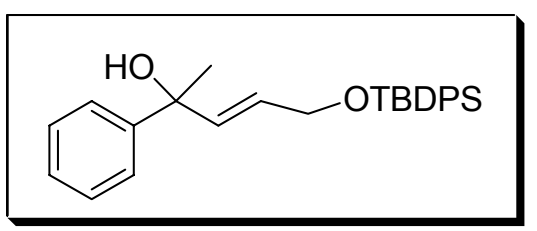

The product was prepared by General Procedure A using $\mathrm{Cp}_{2} \mathrm{ZrHCl}$ (93 mg, $0.36 \mathrm{mmol}$ ), tert-butyldiphenyl-prop-2-ynyloxy-silane (106 mg, $0.36 \mathrm{mmol})$,

$\mathrm{Me}_{2} \mathrm{Zn}(0.18 \mathrm{~mL}, 0.36 \mathrm{mmol}, 2.0 \mathrm{M}$ in toluene), ligand $1(16.4 \mathrm{mg}, 0.03 \mathrm{mmol}, 10 \mathrm{~mol}$ \%), $\mathrm{Me}_{2} \mathrm{Zn}(0.06 \mathrm{~mL}, 0.12 \mathrm{mmol}, 2.0 \mathrm{M}$ in toluene $), \operatorname{Ti}\left(\mathrm{O}^{i} \mathrm{Pr}\right)_{4}(0.25 \mathrm{~mL}, 0.36 \mathrm{mmol}, 1.4$ $\mathrm{M}$ toluene solution) and acetophenone $(35 \mu \mathrm{L}, 0.30 \mathrm{mmol})$. The crude product was purified by column chromatography on deactivated silica gel $\left(\mathrm{Et}_{3} \mathrm{~N} / \mathrm{SiO}_{2}=2.5 \% \mathrm{~V} / \mathrm{V}\right.$, hexanes : EtOAc / 94:6) to give $\mathbf{S 3}\left(115 \mathrm{mg}, 92 \%\right.$ yield, $89 \%$ ee) as an oil: $[\alpha]_{\mathrm{D}}{ }^{20}=-3.6$ (c 2.20, $\left.\mathrm{CHCl}_{3}\right) ;{ }^{1} \mathrm{H}$ NMR $\left(\mathrm{C}_{6} \mathrm{D}_{6}, 500 \mathrm{MHz}\right) \delta 1.16(\mathrm{~s}, 9 \mathrm{H}), 1.45(\mathrm{~s}, 3 \mathrm{H}), 4.17(\mathrm{~d}, J=4.9$ Hz, 2H), 5.72-5.77 (m, 1H), $5.99(\mathrm{~d}, J=15.5 \mathrm{~Hz}, 1 \mathrm{H}), 7.07-7.10(\mathrm{~m}, 1 \mathrm{H}), 7.17-7.24(\mathrm{~m}$, 8H), 7.43-7.44 (m, 2H), 7.73-7.77 (m, 4H) ppm; ${ }^{13} \mathrm{C}\left\{{ }^{1} \mathrm{H}\right\}$ NMR $\left(\mathrm{C}_{6} \mathrm{D}_{6}, 125 \mathrm{MHz}\right) \delta 19.5$, 27.1, 30.1, 64.6, 74.0, 125.6, 127.0, 128.1, 128.4, 130.1, 134.2, 136.1, 137.9, 147.7 ppm; 
IR (film) 3564, 3406, 3069, 3028, 2930, 2856, 1589, 1492, 1472, 1446, 1428, $1377 \mathrm{~cm}^{-1}$; HRMS-CI $m / z$ 439.2053 [(M+Na) ${ }^{+}$; calcd for $\mathrm{C}_{27} \mathrm{H}_{32} \mathrm{O}_{2}$ SiNa: 439.2070].

Preparation of 2,4-diphenyl-but-3-en-2-ol (S4).

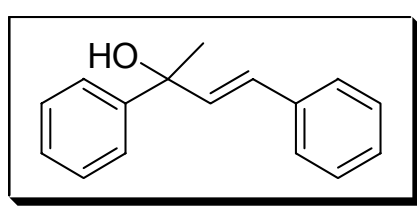

The product was prepared by General Procedure A using $\mathrm{Cp}_{2} \mathrm{ZrHCl}(155 \mathrm{mg}, 0.60 \mathrm{mmol})$, phenylacetylene $(71 \mu \mathrm{L}$, $0.60 \mathrm{mmol}), \mathrm{Me}_{2} \mathrm{Zn}(0.30 \mathrm{~mL}, 0.60 \mathrm{mmol}, 2.0 \mathrm{M}$ in toluene), ligand 1 (27.3 mg, $0.05 \mathrm{mmol}, 10 \mathrm{~mol} \mathrm{\%}), \mathrm{Me}_{2} \mathrm{Zn}(0.10 \mathrm{~mL}, 0.20 \mathrm{mmol}, 2.0 \mathrm{M}$ in toluene), $\operatorname{Ti}\left(\mathrm{O}^{i} \operatorname{Pr}\right)_{4}(0.43 \mathrm{~mL}, 0.60 \mathrm{mmol}, 1.4 \mathrm{M}$ toluene solution) and acetophenone (59 $\mu \mathrm{L}, 0.50 \mathrm{mmol})$. The crude product was purified by column chromatography on deactivated silica gel $\left(\mathrm{Et}_{3} \mathrm{~N} / \mathrm{SiO}_{2}=2.5 \% \mathrm{~V} / \mathrm{V}\right.$, hexanes : EtOAc / 93:7) to give $\mathbf{S 4}(103$ mg, $92 \%$ yield, $87 \%$ ee) as an oil: $[\alpha]_{\mathrm{D}}{ }^{20}=+11.6\left(c\right.$ 2.40, $\left.\mathrm{CHCl}_{3}\right)$. Spectra data for this compound is consistent with the previous charactarization. ${ }^{1}$

\section{Preparation of 4-phenyl-2-(3-trifluoromethyl-phenyl)-but-3-en-2-ol (S5).}

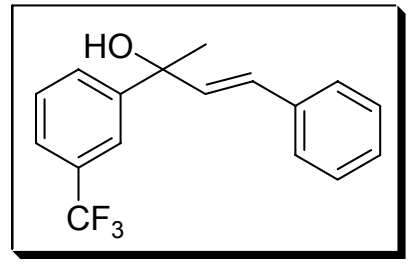

The product was prepared by General Procedure A using $\mathrm{Cp}_{2} \mathrm{ZrHCl}(155 \mathrm{mg}, 0.60 \mathrm{mmol})$, phenylacetylene $(71 \mu \mathrm{L}, 0.60$ $\mathrm{mmol}), \mathrm{Me}_{2} \mathrm{Zn}(0.30 \mathrm{~mL}, 0.60 \mathrm{mmol}, 2.0 \mathrm{M}$ in toluene), ligand 1 (27.3 mg, $0.05 \mathrm{mmol}, 10 \mathrm{~mol} \%), \mathrm{Me}_{2} \mathrm{Zn}(0.10 \mathrm{~mL}, 0.20$ mmol, 2.0 $\mathrm{M}$ in toluene $), \operatorname{Ti}\left(\mathrm{O}^{i} \operatorname{Pr}\right)_{4}(0.43 \mathrm{~mL}, 0.60 \mathrm{mmol}, 1.4 \mathrm{M}$ toluene solution $)$ and 3'(trifluoromethyl)acetophenone $(76 \mu \mathrm{L}, 0.50 \mathrm{mmol})$. The crude product was purified by column chromatography on deactivated silica gel $\left(\mathrm{Et}_{3} \mathrm{~N} / \mathrm{SiO}_{2}=2.5 \% \mathrm{~V} / \mathrm{V}\right.$, hexanes : EtOAc / 93:7) to give S5 (123 mg, 84\% yield, $88 \%$ ee) as an oil: $[\alpha]_{\mathrm{D}}{ }^{20}=+14.8(c 2.33$, 
$\left.\mathrm{CHCl}_{3}\right) ;{ }^{1} \mathrm{H}$ NMR $\left(\mathrm{C}_{6} \mathrm{D}_{6}, 500 \mathrm{MHz}\right) \delta 1.37(\mathrm{~s}, 3 \mathrm{H}), 6.21(\mathrm{~d}, J=16.0 \mathrm{~Hz}, 1 \mathrm{H}), 6.51(\mathrm{~d}, J=$ $16.0 \mathrm{~Hz}, 1 \mathrm{H}), 6.95-7.00(\mathrm{~m}, 1 \mathrm{H}), 7.03-7.16(\mathrm{~m}, 5 \mathrm{H}), 7.28-7.29(\mathrm{~m}, 1 \mathrm{H}), 7.38-7.40(\mathrm{~d}, J=$ $7.8 \mathrm{~Hz}, 1 \mathrm{H}), 7.97(\mathrm{~s}, 1 \mathrm{H}) \mathrm{ppm} ;{ }^{13} \mathrm{C}\left\{{ }^{1} \mathrm{H}\right\} \operatorname{NMR}\left(\mathrm{C}_{6} \mathrm{D}_{6}, 125 \mathrm{MHz}\right) \delta 29.8,74.2,122.3$, 124.0, 127.0, 128.0, 128.6, 128.9, 129.0, 129.4, 130.8, 136.0, 137.0, 148.7 ppm; IR (film) 3272, 3061, 3028, 2980, 2931, 1598, 1494, 1448, $1329 \mathrm{~cm}^{-1}$; HRMS-CI m/z 274.0971 $\left[\left(\mathrm{M}-\mathrm{H}_{2} \mathrm{O}\right)^{+}\right.$; calcd for $\left.\mathrm{C}_{17} \mathrm{H}_{13} \mathrm{~F}_{3}: 274.0970\right]$.

\section{Preparation of 6-(tert-butyl-diphenyl-silanyloxy)-2-(3-trifluoromethyl-phenyl)-hex-} 3-en-2-ol (S6).

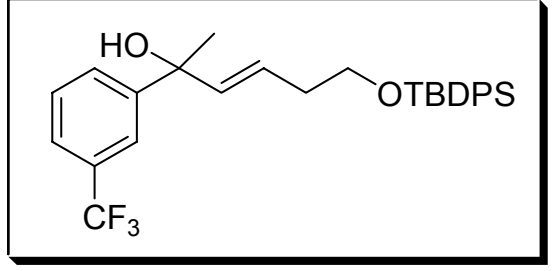

The product was prepared by General Procedure A using $\mathrm{Cp}_{2} \mathrm{ZrHCl}$ (93 mg, $\left.0.36 \mathrm{mmol}\right)$, tert-butyl-but3-ynyloxy-diphenyl-silane (111 mg, $0.36 \mathrm{mmol})$, $\mathrm{Me}_{2} \mathrm{Zn}(0.18 \mathrm{~mL}, 0.36 \mathrm{mmol}, 2.0 \mathrm{M}$ in toluene), ligand 1 (16.4 mg, $0.03 \mathrm{mmol}, 10 \mathrm{~mol} \mathrm{\%}), \mathrm{Me}_{2} \mathrm{Zn}(0.06 \mathrm{~mL}, 0.12 \mathrm{mmol}, 2.0 \mathrm{M}$ in toluene), $\quad \operatorname{Ti}\left(\mathrm{O}^{i} \mathrm{Pr}\right)_{4} \quad(0.25 \mathrm{~mL}, \quad 0.36 \mathrm{mmol}, \quad 1.4 \mathrm{M}$ toluene solution) and 3 '(trifluoromethyl)acetophenone $(46 \mu \mathrm{L}, 0.30 \mathrm{mmol})$. The crude product was purified by column chromatography on deactivated silica gel $\left(\mathrm{Et}_{3} \mathrm{~N} / \mathrm{SiO}_{2}=2.5 \% \mathrm{~V} / \mathrm{V}\right.$, hexanes : EtOAc / 92:8) to give S6 (140 mg, 94\% yield, 90\% ee) as an oil: $[\alpha]_{\mathrm{D}}{ }^{20}=+1.0(c 3.27$, $\left.\mathrm{CHCl}_{3}\right) ;{ }^{1} \mathrm{H}$ NMR $\left(\mathrm{C}_{6} \mathrm{D}_{6}, 500 \mathrm{MHz}\right) \delta 1.14(\mathrm{~s}, 9 \mathrm{H}), 1.33(\mathrm{~s}, 3 \mathrm{H}), 2.13-2.16(\mathrm{~m}, 2 \mathrm{H}), 3.62$ $(\mathrm{t}, J=6.4 \mathrm{~Hz}, 2 \mathrm{H}), 5.56-5.57(\mathrm{~m}, 2 \mathrm{H}), 6.98(\mathrm{t}, J=7.8 \mathrm{~Hz}, 1 \mathrm{H}), 7.23-7.24(\mathrm{~m}, 6 \mathrm{H}), 7.29$ $(\mathrm{d}, \mathrm{J}=7.7 \mathrm{~Hz}, 1 \mathrm{H}), 7.41(\mathrm{~d}, J=7.8 \mathrm{~Hz}, 1 \mathrm{H}), 7.73-7.75(\mathrm{~m}, 4 \mathrm{H}), 7.92(\mathrm{~s}, 1 \mathrm{H}) \mathrm{ppm}$; ${ }^{13} \mathrm{C}\left\{{ }^{1} \mathrm{H}\right\}$ NMR $\left(\mathrm{C}_{6} \mathrm{D}_{6}, 125 \mathrm{MHz}\right) \delta 19.5,27.1,30.0,35.9,63.8,73.9,122.4,123.7,126.2$, 128.1, 128.3, 128.8, 129.3, 130.1, 134.3, 136.0, 138.8, 149.1 ppm; IR (film) 3564, 3408, 
$3071,2931,2858,1668,1590,1488,1472,1428 \mathrm{~cm}^{-1}$; HRMS-CI $\mathrm{m} / z \quad 521.2085$ $\left[(\mathrm{M}+\mathrm{Na})^{+}\right.$; calcd for $\left.\mathrm{C}_{29} \mathrm{H}_{33} \mathrm{~F}_{3} \mathrm{O}_{2} \mathrm{SiNa}: 521.2100\right]$.

\section{Preparation of 5,5-dimethyl-2-m-tolyl-hex-3-en-2-ol (S7)}

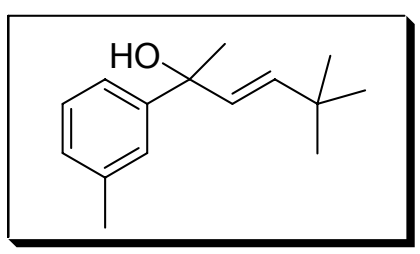

The product was prepared by General Procedure A using $\mathrm{Cp}_{2} \mathrm{ZrHCl}$ (155 mg, $\left.0.60 \mathrm{mmol}\right)$, 3,3-dimethyl-1-butyne (75 $\mu \mathrm{L}, 0.60 \mathrm{mmol}), \mathrm{Me}_{2} \mathrm{Zn}(0.30 \mathrm{~mL}, 0.60 \mathrm{mmol}, 2.0 \mathrm{M}$ in toluene), ligand 1 (27.3 mg, $0.05 \mathrm{mmol}, 10 \mathrm{~mol} \%), \mathrm{Me}_{2} \mathrm{Zn}(0.10 \mathrm{~mL}, 0.20 \mathrm{mmol}, 2.0 \mathrm{M}$ in toluene), $\operatorname{Ti}\left(\mathrm{O}^{i} \mathrm{Pr}\right)_{4}(0.43 \mathrm{~mL}, 0.60 \mathrm{mmol}, 1.4 \mathrm{M}$ toluene solution $)$ and 3'methylacetophenone $(68 \mu \mathrm{L}, 0.50 \mathrm{mmol})$. The crude product was purified by column chromatography on deactivated silica gel $\left(\mathrm{Et}_{3} \mathrm{~N} / \mathrm{SiO}_{2}=2.5 \% \mathrm{~V} / \mathrm{V}\right.$, hexanes : EtOAc / 96:4) to give $\mathbf{S} 7\left(101 \mathrm{mg}, 93 \%\right.$ yield, $92 \%$ ee) as an oil: $[\alpha]_{\mathrm{D}}{ }^{20}=-3.9\left(c 2.33, \mathrm{CHCl}_{3}\right) ;{ }^{1} \mathrm{H}$ NMR $\left(\mathrm{C}_{6} \mathrm{D}_{6}, 500 \mathrm{MHz}\right) \delta 0.97(\mathrm{~s}, 3 \mathrm{H}), 1.55(\mathrm{~s}, 3 \mathrm{H}), 2.18(\mathrm{~s}, 3 \mathrm{H}), 5.72(\mathrm{~d}, J=15.8 \mathrm{~Hz}$, 1H), 5.77 (d, $J=15.8 \mathrm{~Hz}, 1 \mathrm{H}), 6.92(\mathrm{~d}, J=7.4 \mathrm{~Hz}, 1 \mathrm{H}), 7.15(\mathrm{~d}, J=7.7 \mathrm{~Hz}, 1 \mathrm{H}), 7.32(\mathrm{~d}$, $J=7.7 \mathrm{~Hz}, 1 \mathrm{H}), 7.41(\mathrm{~s}, 1 \mathrm{H}) \mathrm{ppm} ;{ }^{13} \mathrm{C}\left\{{ }^{1} \mathrm{H}\right\} \mathrm{NMR}\left(\mathrm{C}_{6} \mathrm{D}_{6}, 125 \mathrm{MHz}\right) \delta 21.7,29.8,30.5$, 32.7, 74.2, 122.9, 126.4, 127.6, 128.4, 133.1, 137.6, 139.0, 148.4 ppm; IR (film) 3382, 3022, 2958, 2865, 1662, 1606, 1588, 1462, $1362 \mathrm{~cm}^{-1}$. HRMS-CI m/z 200.1573 [(M$\left.\mathrm{H}_{2} \mathrm{O}\right)^{+}$; calcd for $\left.\mathrm{C}_{15} \mathrm{H}_{20}: 200.1567\right]$.

\section{Preparation of 8-chloro-2-m-tolyl-oct-3-en-2-ol (S8).}

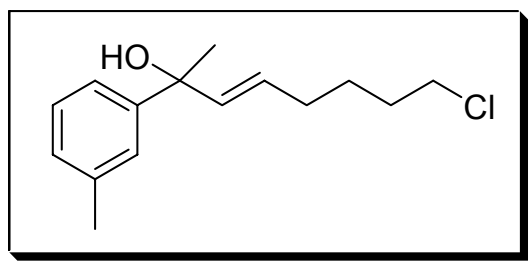

The product was prepared by General Procedure A using $\mathrm{Cp}_{2} \mathrm{ZrHCl}$ (155 mg, $\left.0.60 \mathrm{mmol}\right)$, 6-chloro-hex-1yne $(73 \mu \mathrm{L}, 0.60 \mathrm{mmol}), \mathrm{Me}_{2} \mathrm{Zn}(0.30 \mathrm{~mL}, 0.60 \mathrm{mmol}$, 
2.0 $\mathrm{M}$ in toluene), ligand $1(27.3 \mathrm{mg}, 0.05 \mathrm{mmol}, 10 \mathrm{~mol} \%), \mathrm{Me}_{2} \mathrm{Zn}(0.10 \mathrm{~mL}, 0.20$ mmol, 2.0 $\mathrm{M}$ in toluene), $\mathrm{Ti}\left(\mathrm{O}^{i} \mathrm{Pr}\right)_{4}(0.43 \mathrm{~mL}, 0.60 \mathrm{mmol}, 1.4 \mathrm{M}$ toluene solution $)$ and 3'methylacetophenone $(68 \mu \mathrm{L}, 0.50 \mathrm{mmol})$. The crude product was purified by column chromatography on deactivated silica gel $\left(\mathrm{Et}_{3} \mathrm{~N} / \mathrm{SiO}_{2}=2.5 \% \mathrm{~V} / \mathrm{V}\right.$, hexanes : EtOAc / 93:7) to give $\mathbf{S 8}\left(124 \mathrm{mg}, 98 \%\right.$ yield, $90 \%$ ee) as an oil: $[\alpha]_{\mathrm{D}}{ }^{20}=-0.9\left(c 2.40, \mathrm{CHCl}_{3}\right) ;{ }^{1} \mathrm{H}$ $\operatorname{NMR}\left(\mathrm{C}_{6} \mathrm{D}_{6}, 500 \mathrm{MHz}\right) \delta 1.18-1.24(\mathrm{~m}, 2 \mathrm{H}), 1.38-1.44(\mathrm{~m}, 2 \mathrm{H}), 1.52(\mathrm{~s}, 3 \mathrm{H}), 1.76-1.80$ $(\mathrm{m}, 2 \mathrm{H}), 2.19(\mathrm{~s}, 3 \mathrm{H}), 3.08(\mathrm{t}, J=6.6 \mathrm{~Hz}, 2 \mathrm{H}), 5.50-5.56(\mathrm{~m}, 1 \mathrm{H}), 5.68(\mathrm{~d}, J=15.0 \mathrm{~Hz}$, 1H), $6.93(\mathrm{~d}, J=7.3 \mathrm{~Hz}, 1 \mathrm{H}), 7.16(\mathrm{t}, J=7.5 \mathrm{~Hz}, 1 \mathrm{H}), 7.30(\mathrm{~d}, J=7.7 \mathrm{~Hz}, 1 \mathrm{H}), 7.37(\mathrm{~s}$, 1H) ppm; ${ }^{13} \mathrm{C}\left\{{ }^{1} \mathrm{H}\right\}$ NMR $\left(\mathrm{C}_{6} \mathrm{D}_{6}, 125 \mathrm{MHz}\right) \delta 21.7,26.7,30.3,31.7,32.4,44.8,74.3$, 122.9, 126.4, 127.5, 127.7, 128.4, 137.7, 138.6, 148.1 ppm; IR (film) 3397, 3023, 2931, 2860, 1606, 1588, 1487, 1454, $1369 \mathrm{~cm}^{-1}$; HRMS-CI m/z 234.1179 [(M-H $\left.\mathrm{H}_{2} \mathrm{O}\right)^{+}$; calcd for $\left.\mathrm{C}_{15} \mathrm{H}_{19} \mathrm{Cl}: 234.1176\right]$.

Preparation of 2,4-dimethyl-dec-5-en-4-ol (S9).

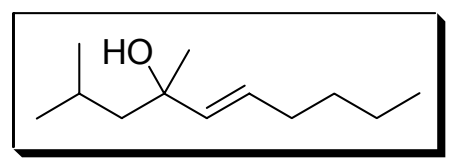

The product was prepared by General Procedure A using $\mathrm{Cp}_{2} \mathrm{ZrHCl}(155 \mathrm{mg}, 0.60 \mathrm{mmol}), 1$-hexyne $(70 \mu \mathrm{L}, 0.60$ $\mathrm{mmol}), \mathrm{Me}_{2} \mathrm{Zn}(0.30 \mathrm{~mL}, 0.60 \mathrm{mmol}, 2.0 \mathrm{M}$ in toluene), ligand 1 (27.3 mg, $0.05 \mathrm{mmol}, 10 \mathrm{~mol} \mathrm{\%}), \mathrm{Me}_{2} \mathrm{Zn}(0.10 \mathrm{~mL}, 0.20 \mathrm{mmol}, 2.0 \mathrm{M}$ in toluene), $\operatorname{Ti}\left(\mathrm{O}^{i} \mathrm{Pr}\right)_{4}(0.43 \mathrm{~mL}, 0.60 \mathrm{mmol}, 1.4 \mathrm{M}$ toluene solution) and 4-methyl-pentan-2one $(62.5 \mu \mathrm{L}, 0.50 \mathrm{mmol})$. The crude product was purified by column chromatography on deactivated silica gel $\left(\mathrm{Et}_{3} \mathrm{~N} / \mathrm{SiO}_{2}=2.5 \% \mathrm{~V} / \mathrm{V}\right.$, hexanes : EtOAc / 96:4) to give $\mathbf{S 9}(78 \mathrm{mg}$, $85 \%$ yield, $79 \%$ ee $)$ as an oil: $[\alpha]_{\mathrm{D}}{ }^{20}=+3.4\left(\right.$ c $\left.2.50, \mathrm{CHCl}_{3}\right) ;{ }^{1} \mathrm{H} \mathrm{NMR}\left(\mathrm{CDCl}_{3}, 500 \mathrm{MHz}\right)$ $\delta 0.88(\mathrm{t}, J=7.1 \mathrm{~Hz}, 3 \mathrm{H}), 0.91(\mathrm{~d}, J=2.9 \mathrm{~Hz}, 3 \mathrm{H}), 0.92(\mathrm{~d}, J=2.9 \mathrm{~Hz}, 3 \mathrm{H}), 1.25(\mathrm{~s}, 3 \mathrm{H})$, 
$1.28-1.39(\mathrm{~m}, 4 \mathrm{H}), 1.40-1.44(\mathrm{~m}, 2 \mathrm{H}), 1.68-1.74(\mathrm{~m}, 1 \mathrm{H}), 2.00-2.04(\mathrm{~m}, 2 \mathrm{H}), 5.51(\mathrm{~d}, J=$ $15.6 \mathrm{~Hz}, 1 \mathrm{H}), 5.55-5.61(\mathrm{~m}, 1 \mathrm{H}) \mathrm{ppm} ;{ }^{13} \mathrm{C}\left\{{ }^{1} \mathrm{H}\right\} \mathrm{NMR}\left(\mathrm{CDCl}_{3}, 125 \mathrm{MHz}\right) \delta 14.3,22.6$, 24.8, 24.9, 25.0, 29.3, 31.9, 32.3, 51.9, 73.6, 127.8, 137.9 ppm; IR (film) 3414, 2955, 2923, 2870, 1668, 1466, $1366 \mathrm{~cm}^{-1}$; HRMS-CI m/z 167.1799 [(M-OH $)^{+}$; calcd for $\mathrm{C}_{12} \mathrm{H}_{23}$ : 167.1800].

\section{Preparation of 3-methyl-1-phenyl-nona-1,4-dien-3-ol (S10).}

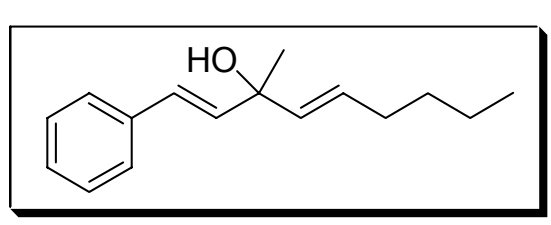

The product was prepared by General Procedure A using $\mathrm{Cp}_{2} \mathrm{ZrHCl}(155 \mathrm{mg}, 0.60 \mathrm{mmol})$, 1-hexyne (70 $\mu \mathrm{L}, 0.60 \mathrm{mmol}), \mathrm{Me}_{2} \mathrm{Zn}(0.30 \mathrm{~mL}, 0.60 \mathrm{mmol}, 2.0 \mathrm{M}$

in toluene), ligand 1 (27.3 mg, $0.05 \mathrm{mmol}, 10 \mathrm{~mol} \%), \mathrm{Me}_{2} \mathrm{Zn}(0.10 \mathrm{~mL}, 0.20 \mathrm{mmol}, 2.0$ $\mathrm{M}$ in toluene $), \operatorname{Ti}\left(\mathrm{O}^{i} \mathrm{Pr}\right)_{4}(0.43 \mathrm{~mL}, 0.60 \mathrm{mmol}, 1.4 \mathrm{M}$ toluene solution $)$ and trans-4phenyl-3-buten-2-one $(73 \mathrm{mg}, 0.50 \mathrm{mmol})$. The crude product was purified by column chromatography on deactivated silica gel $\left(\mathrm{Et}_{3} \mathrm{~N} / \mathrm{SiO}_{2}=2.5 \% \mathrm{~V} / \mathrm{V}\right.$, hexanes : EtOAc / 95:5) to give $\mathbf{S 1 0}\left(100 \mathrm{mg}, 87 \%\right.$ yield, $92 \%$ ee) as an oil: $[\alpha]_{\mathrm{D}}{ }^{20}=-12.4\left(c 2.48, \mathrm{CHCl}_{3}\right)$; ${ }^{1} \mathrm{H}$ NMR $\left(\mathrm{C}_{6} \mathrm{D}_{6}, 500 \mathrm{MHz}\right) \delta 0.86(\mathrm{t}, J=7.1 \mathrm{~Hz}, 3 \mathrm{H}), 1.23-1.30(\mathrm{~m}, 4 \mathrm{H}), 1.39(\mathrm{~s}, 3 \mathrm{H})$, 1.95-2.00 (m, 2H), 5.59-5.63 (m, 1H), 5.67-5.73 (m, 1H), $6.30(\mathrm{~d}, J=16.0 \mathrm{~Hz}, 1 \mathrm{H}), 6.68$ $(\mathrm{d}, J=16.0 \mathrm{~Hz}, 1 \mathrm{H}), 7.03-7.06(\mathrm{~m}, 1 \mathrm{H}), 7.11-7.14(\mathrm{~m}, 2 \mathrm{H}), 7.27-7.299 \mathrm{~m}, 2 \mathrm{H}) \mathrm{ppm}$; ${ }^{13} \mathrm{C}\left\{{ }^{1} \mathrm{H}\right\}$ NMR $\left(\mathrm{C}_{6} \mathrm{D}_{6}, 125 \mathrm{MHz}\right) \delta 14.2,22.7,28.9,31.9,32.4,73.0,126.9,127.5,127.6$, 128.8, 128.9, 136.7, 136.7, 137.7 ppm; IR (film) 3366, 3025, 2957, 2928, 2856, 1664, 1599, 1578, 1494, $1448 \mathrm{~cm}^{-1}$; HRMS-CI m/z $230.1674\left[\mathrm{M}^{+}\right.$; calcd for $\mathrm{C}_{16} \mathrm{H}_{22} \mathrm{O}$ : 230.1671]. 


\section{Preparation of 1-hex-1-enyl-2,4,4-trimethyl-cyclohex-2-enol (S11).}

The product was prepared by General Procedure A using

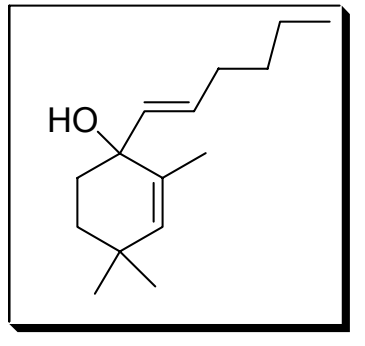

$\mathrm{Cp}_{2} \mathrm{ZrHCl}(155 \mathrm{mg}, 0.60 \mathrm{mmol}), 1$-hexyne $(70 \mu \mathrm{L}, 0.60 \mathrm{mmol})$, $\mathrm{Me}_{2} \mathrm{Zn}(0.30 \mathrm{~mL}, 0.60 \mathrm{mmol}, 2.0 \mathrm{M}$ in toluene), ligand $1(27.3 \mathrm{mg}$, $0.05 \mathrm{mmol}, 10 \mathrm{~mol} \%), \mathrm{Me}_{2} \mathrm{Zn}(0.10 \mathrm{~mL}, 0.20 \mathrm{mmol}, 2.0 \mathrm{M}$ in toluene), $\operatorname{Ti}\left(\mathrm{O}^{i} \mathrm{Pr}\right)_{4}(0.43 \mathrm{~mL}, 0.60 \mathrm{mmol}, 1.4 \mathrm{M}$ toluene solution $)$ and 2,4,4-trimethyl-2-cyclohexen-1-one $(75 \mu \mathrm{L}, 0.50 \mathrm{mmol})$. The crude product was purified by column chromatography on deactivated silica gel $\left(\mathrm{Et}_{3} \mathrm{~N} / \mathrm{SiO}_{2}=2.5 \% \mathrm{~V} / \mathrm{V}\right.$, hexanes : EtOAc / 95:5) to give S11 (104 mg, 94\% yield, 97\% ee) as an oil: $[\alpha]_{\mathrm{D}}{ }^{20}=-$ $72.0\left(c 0.60, \mathrm{CHCl}_{3}\right) ;{ }^{1} \mathrm{H} \mathrm{NMR}\left(\mathrm{CDCl}_{3}, 500 \mathrm{MHz}\right) \delta 0.88(\mathrm{t}, J=7.0 \mathrm{~Hz}, 3 \mathrm{H}), 0.95(\mathrm{~s}, 3 \mathrm{H})$, $0.99(\mathrm{~s}, 3 \mathrm{H}), 1.28-1.35(\mathrm{~m}, 4 \mathrm{H}), 1.36-1.39(\mathrm{~m}, 2 \mathrm{H}), 1.61(\mathrm{~s}, 3 \mathrm{H}), 1.73-1.77(\mathrm{~m}, 2 \mathrm{H}), 2.06-$ $2.07(\mathrm{~m}, 2 \mathrm{H}), 5.24(\mathrm{~s}, 1 \mathrm{H}), 5.41(\mathrm{~d}, J=15.5 \mathrm{~Hz}, 1 \mathrm{H}), 5.53-5.59(\mathrm{~m}, 1 \mathrm{H}) \mathrm{ppm} ;{ }^{13} \mathrm{C}\left\{{ }^{1} \mathrm{H}\right\}$ $\operatorname{NMR}\left(\mathrm{CDCl}_{3}, 125 \mathrm{MHz}\right) \delta 14.3,18.4,22.6,29.6,30.2,32.0,32.3,32.6,34.2,36.7,74.2$, 130.4, 134.2, 134.8, 136.6 ppm; IR (film) 3378, 2954, 2925, 2858, 2359, 1668, 1451, 1377, $1359 \mathrm{~cm}^{-1}$; HRMS-CI $\mathrm{m} / z 222.1980$ [ $\mathrm{M}^{+}$; calcd for $\left.\mathrm{C}_{15} \mathrm{H}_{26} \mathrm{O}: 222.1984\right]$.

Preparation of 1-[4-(tert-butyl-diphenyl-silanyloxy)-but-1-enyl]-2,4,4-trimethylcyclohex-enol (S12).

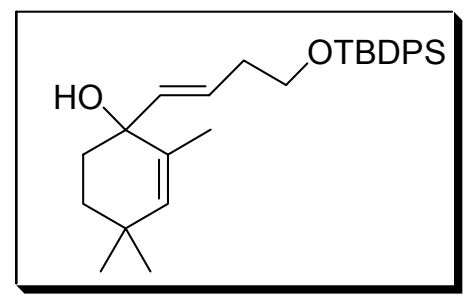

The product was prepared by General Procedure A using $\mathrm{Cp}_{2} \mathrm{ZrHCl}$ (93 mg, $\left.0.36 \mathrm{mmol}\right)$, tert-butyl-but-3-ynyloxydiphenyl-silane (111 mg, $0.36 \mathrm{mmol}), \mathrm{Me}_{2} \mathrm{Zn}(0.18 \mathrm{~mL}$, $0.36 \mathrm{mmol}, 2.0 \mathrm{M}$ in toluene), ligand $1(16.4 \mathrm{mg}, 0.03$ mmol, $10 \mathrm{~mol} \%), \mathrm{Me}_{2} \mathrm{Zn}(0.06 \mathrm{~mL}, 0.12 \mathrm{mmol}, 2.0 \mathrm{M}$ in toluene $), \operatorname{Ti}\left(\mathrm{O}^{i} \operatorname{Pr}\right)_{4}(0.25 \mathrm{~mL}$, 
$0.36 \mathrm{mmol}, 1.4 \mathrm{M}$ toluene solution) and 2,4,4-trimethyl-2-cyclohexen-1-one (45 $\mu \mathrm{L}, 0.30$ mmol). The crude product was purified by column chromatography on deactivated silica gel $\left(\mathrm{Et}_{3} \mathrm{~N} / \mathrm{SiO}_{2}=2.5 \% \mathrm{~V} / \mathrm{V}\right.$, hexanes : EtOAc / 95:5) to give $\mathbf{S 1 2}(115 \mathrm{mg}, 85 \%$ yield, 94\% ee) as an oil: $[\alpha]_{\mathrm{D}}{ }^{20}=-43.8\left(\right.$ c 3.10, $\left.\mathrm{CHCl}_{3}\right) ;{ }^{1} \mathrm{H}$ NMR $\left(\mathrm{C}_{6} \mathrm{D}_{6}, 500 \mathrm{MHz}\right) \delta 0.92$ (s, $3 \mathrm{H}), 0.93(\mathrm{~s}, 3 \mathrm{H}), 1.17(\mathrm{~s}, 9 \mathrm{H}), 1.44-1.46(\mathrm{~m}, 2 \mathrm{H}), 1.67-1.79(5 \mathrm{H}), 2.25-2.28(\mathrm{~m}, 2 \mathrm{H})$, $3.69(\mathrm{t}, J=6.5 \mathrm{~Hz}, 2 \mathrm{H}), 5.18(\mathrm{~s}, 1 \mathrm{H}), 5.51(\mathrm{~d}, \mathrm{~J}=15.5 \mathrm{~Hz}, 1 \mathrm{H}), 5.69-5.74(\mathrm{~m}, 1 \mathrm{H}), 7.23-$ 7.26 (m, 6H), 7.76-7.79 (m, 4H) ppm; ${ }^{13} \mathrm{C}\left\{{ }^{1} \mathrm{H}\right\}$ NMR $\left(\mathrm{C}_{6} \mathrm{D}_{6}, 125 \mathrm{MHz}\right) \delta 18.4,19.5,27.2$, $29.4,30.1,32.4,34.2,35.8,36.1,64.2,73.6,126.2,128.1,130.0,134.4,134.9,135.8$, 136.1, 137.6 ppm; IR (film) 3397, 3070, 3049, 2953, 1662, 1589, 1568, 1471, $1428 \mathrm{~cm}^{-1}$; HRMS-CI $m / z 471.2696$ [(M+Na) ${ }^{+}$; calcd for $\mathrm{C}_{29} \mathrm{H}_{40} \mathrm{O}_{2} \mathrm{SiNa}$ : 471.2696].

Preparation of 5-(tert-butyl-diphenyl-silanyloxy)-2-cyclohex-1-enyl-pent-3-en-2-ol (S13).

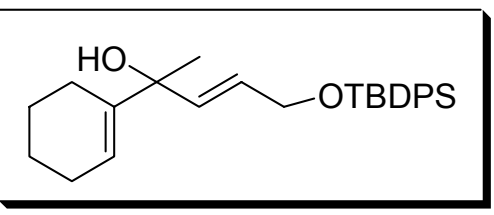

The product was prepared by General Procedure A using $\mathrm{Cp}_{2} \mathrm{ZrHCl}$ (93 mg, $\left.0.36 \mathrm{mmol}\right)$, tert-butyl-diphenylprop-2-ynyloxy-silane (106 mg, $0.36 \mathrm{mmol}), \mathrm{Me}_{2} \mathrm{Zn}$

(0.18 mL, $0.36 \mathrm{mmol}, 2.0 \mathrm{M}$ in toluene), ligand 1 (16.4 mg, $0.03 \mathrm{mmol}, 10 \mathrm{~mol} \%)$, $\mathrm{Me}_{2} \mathrm{Zn}(0.06 \mathrm{~mL}, 0.12 \mathrm{mmol}, 2.0 \mathrm{M}$ in toluene $), \operatorname{Ti}\left(\mathrm{O}^{i} \mathrm{Pr}\right)_{4}(0.25 \mathrm{~mL}, 0.36 \mathrm{mmol}, 1.4 \mathrm{M}$ toluene solution) and 1-cyclohex-1-enyl-ethanone (39 $\mu \mathrm{L}, 0.30 \mathrm{mmol})$. The crude product was purified by column chromatography on deactivated silica gel $\left(\mathrm{Et}_{3} \mathrm{~N} / \mathrm{SiO}_{2}=2.5 \% \mathrm{~V} / \mathrm{V}\right.$, hexanes : EtOAc / 92:8) to give $\mathbf{S 1 3}\left(123 \mathrm{mg}, 98 \%\right.$ yield, $92 \%$ ee) as an oil: $[\alpha]_{\mathrm{D}}{ }^{20}=-1.5$ (c 3.07, $\left.\mathrm{CHCl}_{3}\right) ;{ }^{1} \mathrm{H}$ NMR $\left(\mathrm{C}_{6} \mathrm{D}_{6}, 500 \mathrm{MHz}\right) \delta 1.17(\mathrm{~s}, 9 \mathrm{H}), 1.28(\mathrm{~s}, 3 \mathrm{H}), 1.45-1.47(\mathrm{~m}$, $2 \mathrm{H}), 1.52-1.54(\mathrm{~m}, 2 \mathrm{H}), 1.94-1.97(\mathrm{~m}, 2 \mathrm{H}), 2.00-2.01(\mathrm{~m}, 2 \mathrm{H}), 4.22(\mathrm{~d}, J=3.5 \mathrm{~Hz}, 2 \mathrm{H})$, 
5.74-5.84 (m, 3H), 7.21-7.26 (m, 6H), 7.75-7.80 (m, 4H) ppm; ${ }^{13} \mathrm{C}\left\{{ }^{1} \mathrm{H}\right\}$ NMR $\left(\mathrm{C}_{6} \mathrm{D}_{6}, 125\right.$ MHz) $\delta 19.6,22.8,23.5,24.6,25.6,27.1,27.2,64.7,74.7,120.6,126.7,128.1,130.0$, 134.4, 136.0, 137.2, 142.6 ppm; IR (film) 3564, 3377, 3069, 3047, 2920, 2837, 1447, 1436, 1427, $1376 \mathrm{~cm}^{-1}$; HRMS-CI $\mathrm{m} / z$ 402.2372 $\left[\left(\mathrm{M}-\mathrm{H}_{2} \mathrm{O}\right)^{+}\right.$; calcd for $\mathrm{C}_{27} \mathrm{H}_{34} \mathrm{OSi}$ : 402.2380].

\section{Preparation of 3-phenyl-non-4-en-3-ol (S14).}

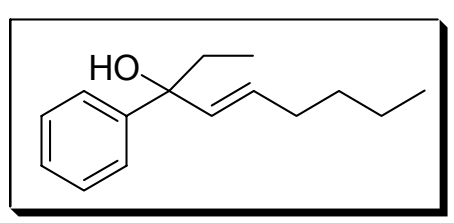

The product was prepared by General Procedure A using $\mathrm{Cp}_{2} \mathrm{ZrHCl}(155 \mathrm{mg}, 0.60 \mathrm{mmol}), 1$-hexyne $(70 \mu \mathrm{L}, 0.60$ $\mathrm{mmol}), \mathrm{Me}_{2} \mathrm{Zn}(0.30 \mathrm{~mL}, 0.60 \mathrm{mmol}, 2.0 \mathrm{M}$ in toluene), ligand $1(27.3 \mathrm{mg}, 0.05 \mathrm{mmol}, 10 \mathrm{~mol} \mathrm{\%}), \mathrm{Me}_{2} \mathrm{Zn}(0.10 \mathrm{~mL}, 0.20 \mathrm{mmol}, 2.0 \mathrm{M}$ in toluene), $\operatorname{Ti}\left(\mathrm{O}^{i} \mathrm{Pr}\right)_{4}(0.43 \mathrm{~mL}, 0.60 \mathrm{mmol}, 1.4 \mathrm{M}$ toluene solution) and propiophenone $(67$ $\mu \mathrm{L}, 0.50 \mathrm{mmol})$. The crude product was purified by column chromatography on deactivated silica gel $\left(\mathrm{Et}_{3} \mathrm{~N} / \mathrm{SiO}_{2}=2.5 \% \mathrm{~V} / \mathrm{V}\right.$, hexanes : EtOAc / 97:3) to give $\mathbf{S 1 4}(98$ mg, $90 \%$ yield, $94 \%$ ee $)$ as an oil: $[\alpha]_{\mathrm{D}}{ }^{20}=-11.0\left(\right.$ c 2.13, $\left.\mathrm{CHCl}_{3}\right) ;{ }^{1} \mathrm{H}$ NMR $\left(\mathrm{C}_{6} \mathrm{D}_{6}, 500\right.$ MHz) $\delta$ 0.82-0.85 (m, 6H), 1.19-1.25 (m, 4H), 1.72-1.78 (m, 1H), 1.79-1.88 (m, 1H), $1.92-1.96(\mathrm{~m}, 2 \mathrm{H}), 5.58-5.64(\mathrm{~m}, 1 \mathrm{H}), 5.73(\mathrm{~d}, J=15.5 \mathrm{~Hz}, 1 \mathrm{H}), 7.09(\mathrm{t}, J=7.3 \mathrm{~Hz}, 1 \mathrm{H})$, $7.21(\mathrm{t}, J=7.5 \mathrm{~Hz}, 2 \mathrm{H}), 7.46(\mathrm{~d}, J=7.3 \mathrm{~Hz}, 2 \mathrm{H}), \mathrm{ppm} ;{ }^{13} \mathrm{C}\left\{{ }^{1} \mathrm{H}\right\}$ NMR $\left(\mathrm{C}_{6} \mathrm{D}_{6}, 125 \mathrm{MHz}\right)$ $\delta 8.2,14.2,22.6,31.9,32.4,35.6,76.7,126.0,126.7,128.3,129.0,137.2,147.0$ ppm; IR (film) 3459, 3024, 2953, 2916, 2872, 1665, 1601, 1493, 1466, $1446 \mathrm{~cm}^{-1}$; HRMS-CI m/z $200.1567\left[\left(\mathrm{M}-\mathrm{H}_{2} \mathrm{O}\right)^{+}\right.$; calcd for $\left.\mathrm{C}_{15} \mathrm{H}_{20}: 200.1565\right]$.

\section{Preparation of 3-(3-chloro-phenyl)-non-4-en-3-ol (S15).}




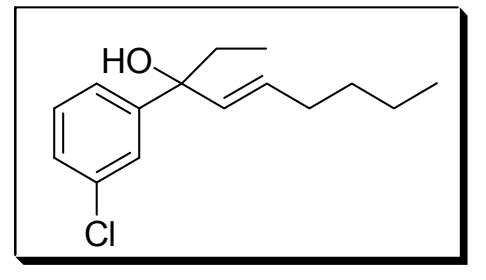

The product was prepared by General Procedure A using $\mathrm{Cp}_{2} \mathrm{ZrHCl}(155 \mathrm{mg}, 0.60 \mathrm{mmol}), 1$-hexyne $(70 \mu \mathrm{L}, 0.60$ $\mathrm{mmol}), \mathrm{Me}_{2} \mathrm{Zn}(0.30 \mathrm{~mL}, 0.60 \mathrm{mmol}, 2.0 \mathrm{M}$ in toluene), ligand 1 (27.3 mg, $0.05 \mathrm{mmol}, 10 \mathrm{~mol} \%), \mathrm{Me}_{2} \mathrm{Zn}(0.10 \mathrm{~mL}$, $0.20 \mathrm{mmol}, 2.0 \mathrm{M}$ in toluene $), \operatorname{Ti}\left(\mathrm{O}^{i} \operatorname{Pr}\right)_{4}(0.43 \mathrm{~mL}, 0.60 \mathrm{mmol}, 1.4 \mathrm{M}$ toluene solution $)$ and 3-chloropropiophenone ( $84 \mathrm{mg}, 0.50 \mathrm{mmol}$ ). The crude product was purified by column chromatography on deactivated silica gel $\left(\mathrm{Et}_{3} \mathrm{~N} / \mathrm{SiO}_{2}=2.5 \% \mathrm{~V} / \mathrm{V}\right.$, hexanes : EtOAc / 97:3) to give S15 (117 mg, 93\% yield, 93\% ee) as an oil: $[\alpha]_{\mathrm{D}}{ }^{20}=-3.2(c 2.02$, $\left.\mathrm{CHCl}_{3}\right) ;{ }^{1} \mathrm{H}$ NMR $\left(\mathrm{C}_{6} \mathrm{D}_{6}, 500 \mathrm{MHz}\right) \delta 0.74(\mathrm{t}, J=7.4 \mathrm{~Hz}, 3 \mathrm{H}), 0.84(\mathrm{t}, J=7.0 \mathrm{~Hz}, 3 \mathrm{H})$, $1.20-1.25(\mathrm{~m}, 4 \mathrm{H}), 1.56-1.71(\mathrm{~m}, 2 \mathrm{H}), 1.87-1.91(\mathrm{~m}, 2 \mathrm{H}), 5.50-5.57(\mathrm{~m}, 2 \mathrm{H}), 6.90(\mathrm{t}, J=$ $7.9 \mathrm{~Hz}, 1 \mathrm{H}), 7.05(\mathrm{~m}, 1 \mathrm{H}), 7.16(\mathrm{~d}, J=7.7 \mathrm{~Hz}, 1 \mathrm{H}), 7.59(\mathrm{t}, J=1.8 \mathrm{~Hz}, 1 \mathrm{H}) \mathrm{ppm} ;{ }^{13} \mathrm{C}\left\{{ }^{1} \mathrm{H}\right\}$ NMR $\left(\mathrm{C}_{6} \mathrm{D}_{6}, 125 \mathrm{MHz}\right) \delta 8.0,14.1,22.6,31.8,32.3,35.4,76.4,124.2,126.4,126.9$, 129.5, 129.6, 134.5, 136.7, 149.4 ppm; IR (film) 3455, 3024, 2958, 2927, 2872, 1665 , 1595, 1572, 1466, $1420 \mathrm{~cm}^{-1}$; HRMS-CI $m / z 234.1175\left[\left(\mathrm{M}-\mathrm{H}_{2} \mathrm{O}\right)^{+}\right.$; calcd for $\mathrm{C}_{15} \mathrm{H}_{19} \mathrm{Cl}$ : 234.1175].

\section{Conditions for the Determination of Enantiomeric Excess.}

The allylic alcohols S1-S2, S7, S9, S11 and S14-S15 were analyzed by chiral capillary GC. The specifications for the GC analyses were as follows: Fused silica chiral capillary column (Supelco $\beta$-Dex 120): $30 \mathrm{~m}$ x $0.25 \mathrm{~mm}$ (id) x $0.25 \mu \mathrm{m}$ film thickness. Carrier gas: 
nitrogen. Inlet temperature: $250{ }^{\circ} \mathrm{C}$. Detector: FID, $270{ }^{\circ} \mathrm{C}$. The conditions for the resolution of the racemates by $\mathrm{GC}$ are given below.

2-Phenyl-oct-3-en-2-ol (S1). $\mathrm{t}_{1}=72.6 \mathrm{~min}, \mathrm{t}_{2}=77.1 \mathrm{~min}\left(130^{\circ} \mathrm{C}, 1.0 \mathrm{~mL} / \mathrm{min}\right)$.

4-Cyclopropyl-2-phenyl-but-3-en-2-ol (S2). $\mathrm{t}_{1}=34.9 \mathrm{~min}, \mathrm{t}_{2}=35.7 \min \left(140^{\circ} \mathrm{C}, 1.0\right.$ $\mathrm{mL} / \mathrm{min})$.

5,5-Dimethyl-2-m-tolyl-hex-3-en-2-ol (S7). $\mathrm{t}_{1}=51.9 \mathrm{~min}, \mathrm{t}_{2}=55.0 \min \left(125^{\circ} \mathrm{C}, 1.5\right.$ $\mathrm{mL} / \mathrm{min})$.

2,4-Dimethyl-dec-5-en-4-ol (S9). $\mathrm{t}_{1}=13.4 \mathrm{~min}, \mathrm{t}_{2}=14.2 \mathrm{~min}\left(120^{\circ} \mathrm{C}, 1.0 \mathrm{~mL} / \mathrm{min}\right)$.

1-Hex-1-enyl-2,4,4-trimethyl-cyclohex-2-enol (S11). $\mathrm{t}_{1}=13.6 \mathrm{~min}, \mathrm{t}_{2}=14.4 \mathrm{~min}$ $\left(145^{\circ} \mathrm{C}, 1.0 \mathrm{~mL} / \mathrm{min}\right)$.

3-Phenyl-non-4-en-3-ol (S14). $\mathrm{t}_{1}=25.6 \mathrm{~min}, \mathrm{t}_{2}=26.6 \mathrm{~min}\left(130^{\circ} \mathrm{C}, 1.0 \mathrm{~mL} / \mathrm{min}\right)$.

3-(3-Chloro-phenyl)-non-4-en-3-ol (S15). $\mathrm{t}_{1}=54.6 \mathrm{~min}, \mathrm{t}_{2}=56.5 \min \left(135^{\circ} \mathrm{C}, 1.0\right.$ $\mathrm{mL} / \mathrm{min})$.

The analysis of S3-S6, S8, S10 and S12-S13 was performed by chiral HPLC using a Chiralcel OD-H column. The conditions for the resolution are described below.

5-(tert-Butyl-diphenyl-silanyloxy)-2-phenyl-pent-3-en-2-ol (S3). $\mathrm{t}_{1}=30.0 \mathrm{~min}, \mathrm{t}_{2}=32.7$ $\min$ (hexane /2-propanol : 98 / 2, $0.3 \mathrm{~mL} / \mathrm{min}$ ).

2,4-Diphenyl-but-3-en-2-ol (S4). $\mathrm{t}_{1}=12.2 \mathrm{~min}, \mathrm{t}_{2}=15.6 \mathrm{~min}$ (hexane $/ 2$-propanol : $92 / 8$, $0.8 \mathrm{~mL} / \mathrm{min})$. 
4-Phenyl-2-(3-trifluoromethyl-phenyl)-but-3-en-2-ol (S5). $\mathrm{t}_{1}=19.4 \mathrm{~min}, \mathrm{t}_{2}=30.8 \mathrm{~min}$ (hexane /2-propanol : $92 / 8,0.5 \mathrm{~mL} / \mathrm{min}$ ).

6-(tert-Butyl-diphenyl-silanyloxy)-2-(3-trifluoromethyl-phenyl)-hex-3-en-2-ol (S6).

$\mathrm{t}_{1}=28.0 \mathrm{~min}, \mathrm{t}_{2}=30.8 \mathrm{~min}$ (hexane $/ 2$-propanol : $\left.99.5 / 0.5,0.5 \mathrm{~mL} / \mathrm{min}\right)$.

8-Chloro-2-m-tolyl-oct-3-en-2-ol (S8). $\mathrm{t}_{1}=17.7 \mathrm{~min}, \mathrm{t}_{2}=19.0 \mathrm{~min}$ (hexane $/ 2$-propanol : 97 / 3, $0.5 \mathrm{~mL} / \mathrm{min})$.

3-Methyl-1-phenyl-nona-1,4-dien-3-ol (S10). $\mathrm{t}_{1}=24.8 \mathrm{~min}, \mathrm{t}_{2}=28.7 \mathrm{~min}$ (hexane $/ 2$ propanol : $97 / 3,0.5 \mathrm{~mL} / \mathrm{min})$.

1-[4-(tert-Butyl-diphenyl-silanyloxy)-but-1-enyl]-2,4,4-trimethyl-cyclohex-enol (S12). $\mathrm{t}_{1}=8.6 \mathrm{~min}, \mathrm{t}_{2}=11.0 \mathrm{~min}$ (hexane $/ 2$-propanol : $\left.97 / 3,0.5 \mathrm{~mL} / \mathrm{min}\right)$.

5-(tert-Butyl-diphenyl-silanyloxy)-2-cyclohex-1-enyl-pent-3-en-2-ol (S13). $t_{1}=20.5$ $\min , \mathrm{t}_{2}=21.7 \mathrm{~min}$ (hexane $/ 2$-propanol : $\left.98 / 2,0.3 \mathrm{~mL} / \mathrm{min}\right)$.

\section{Reference}

1) Garcia C.; Walsh P. J. Org. Lett. 2003, 3641-3644. 


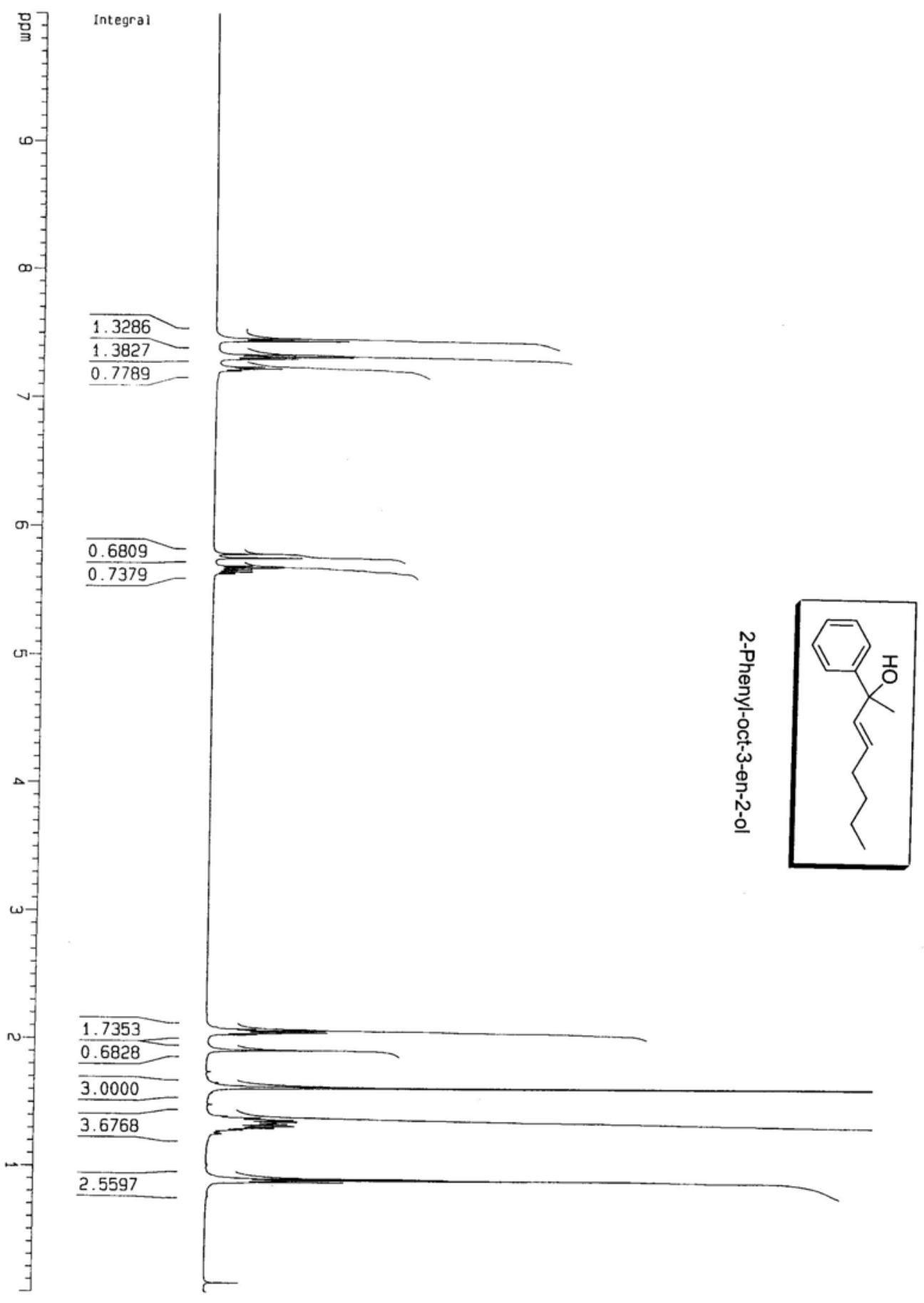



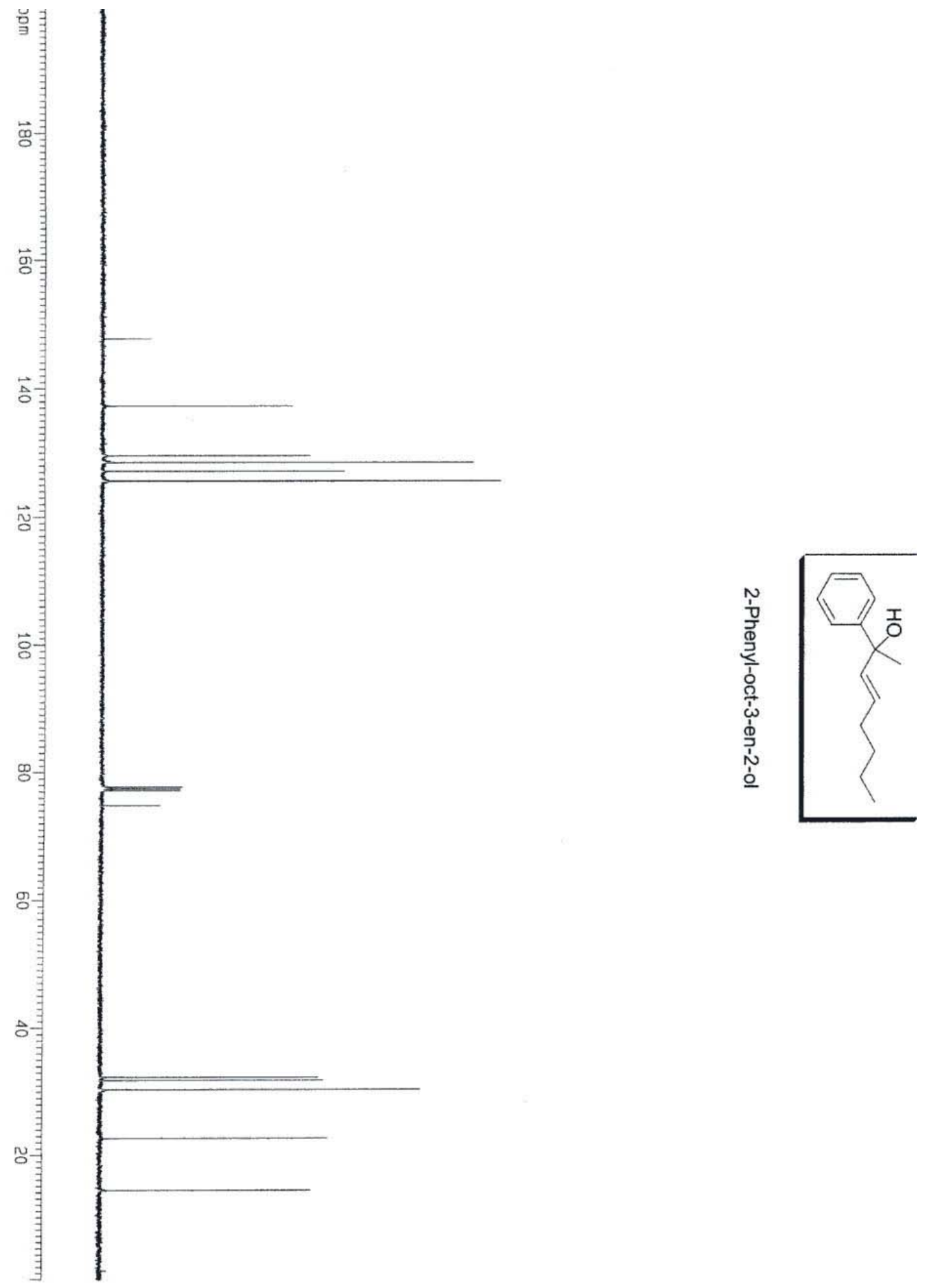


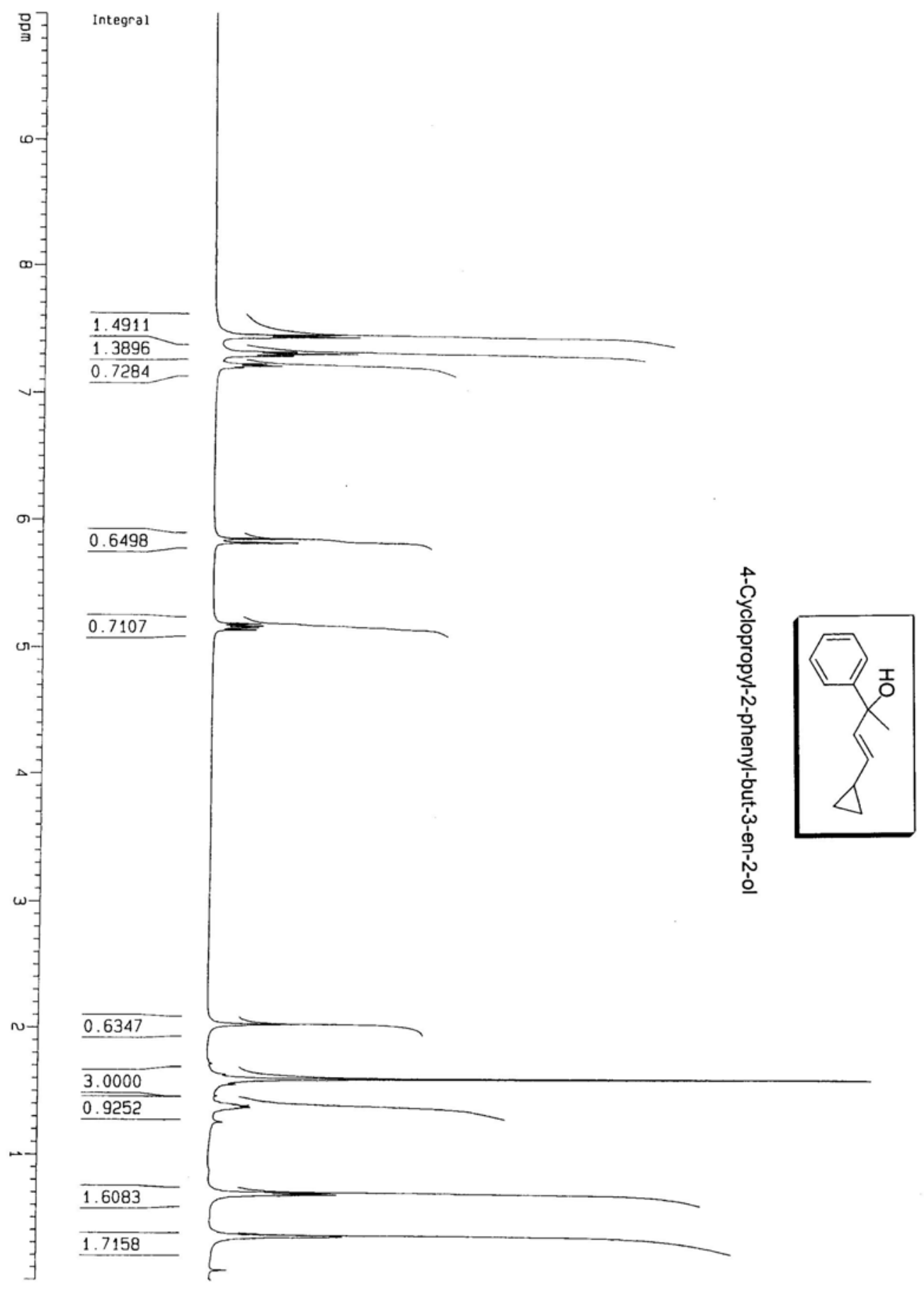



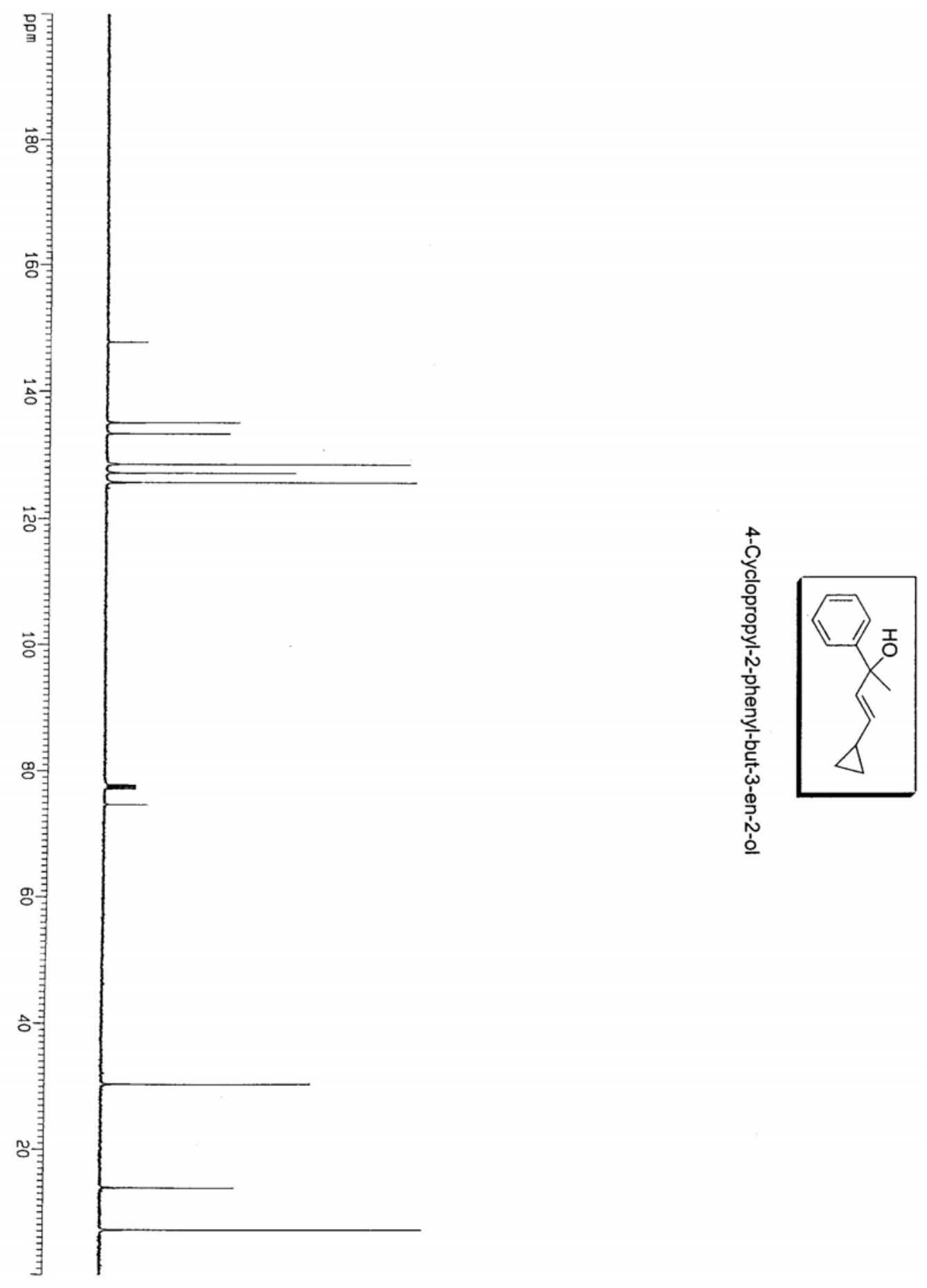


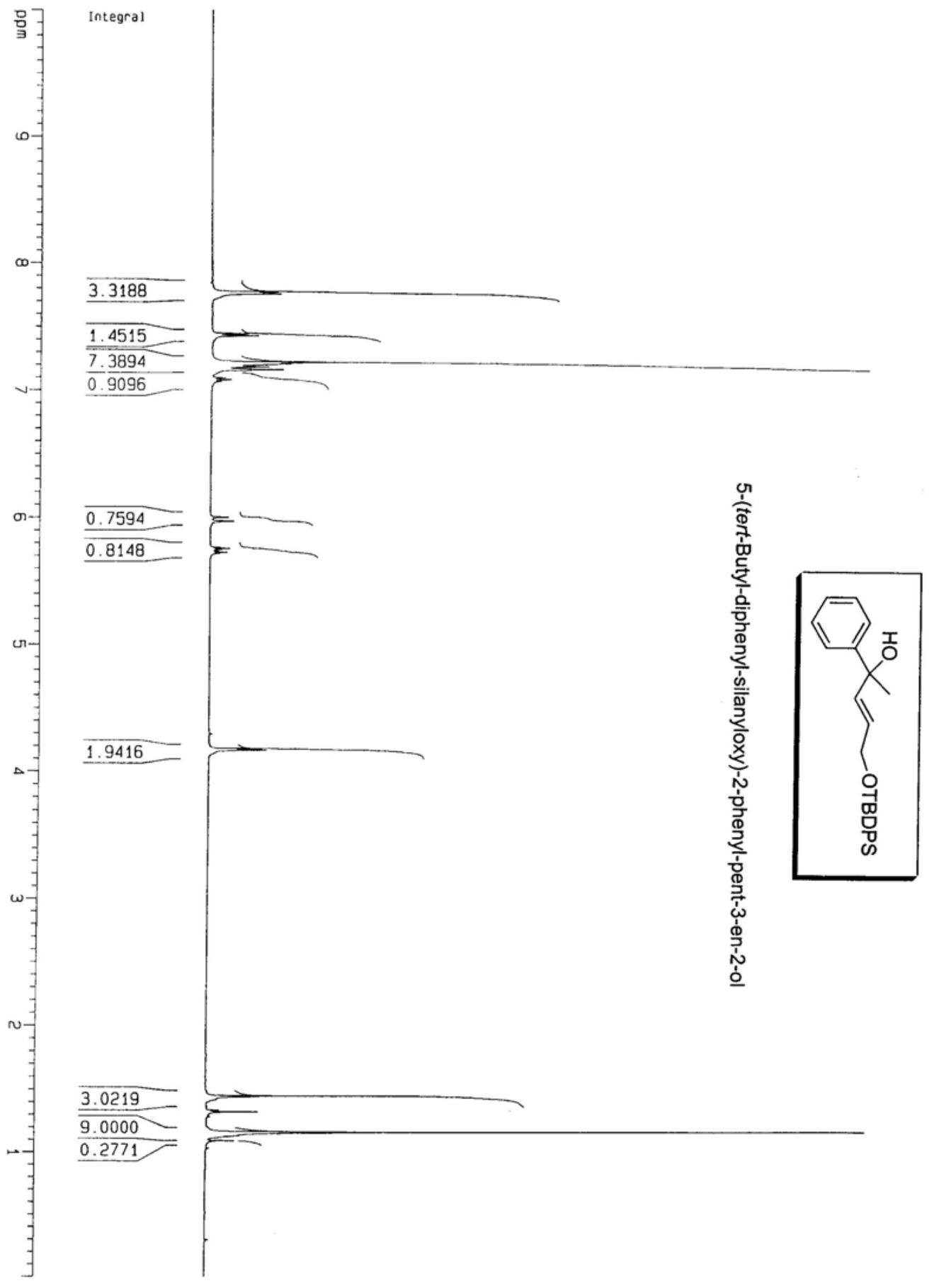




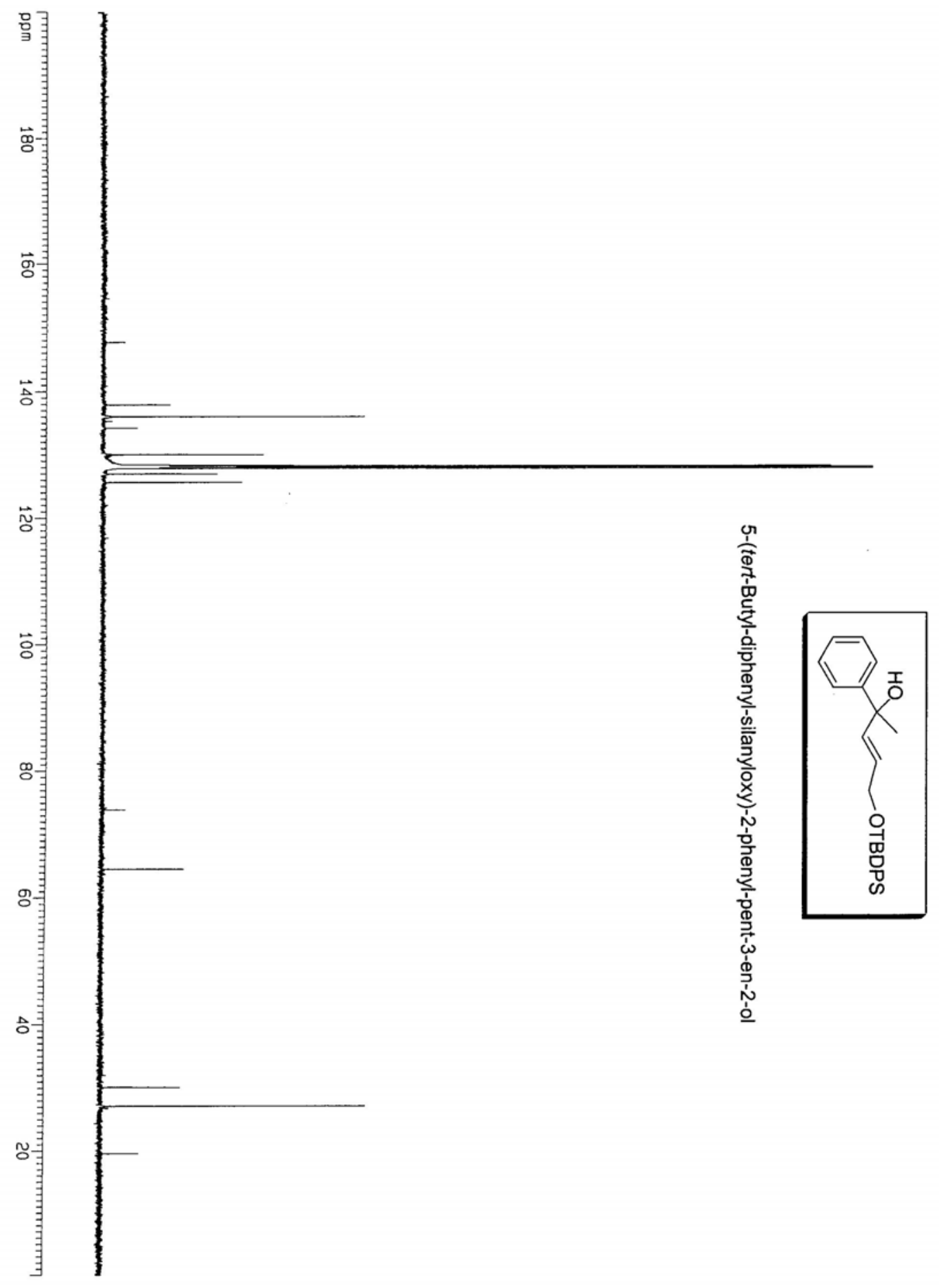




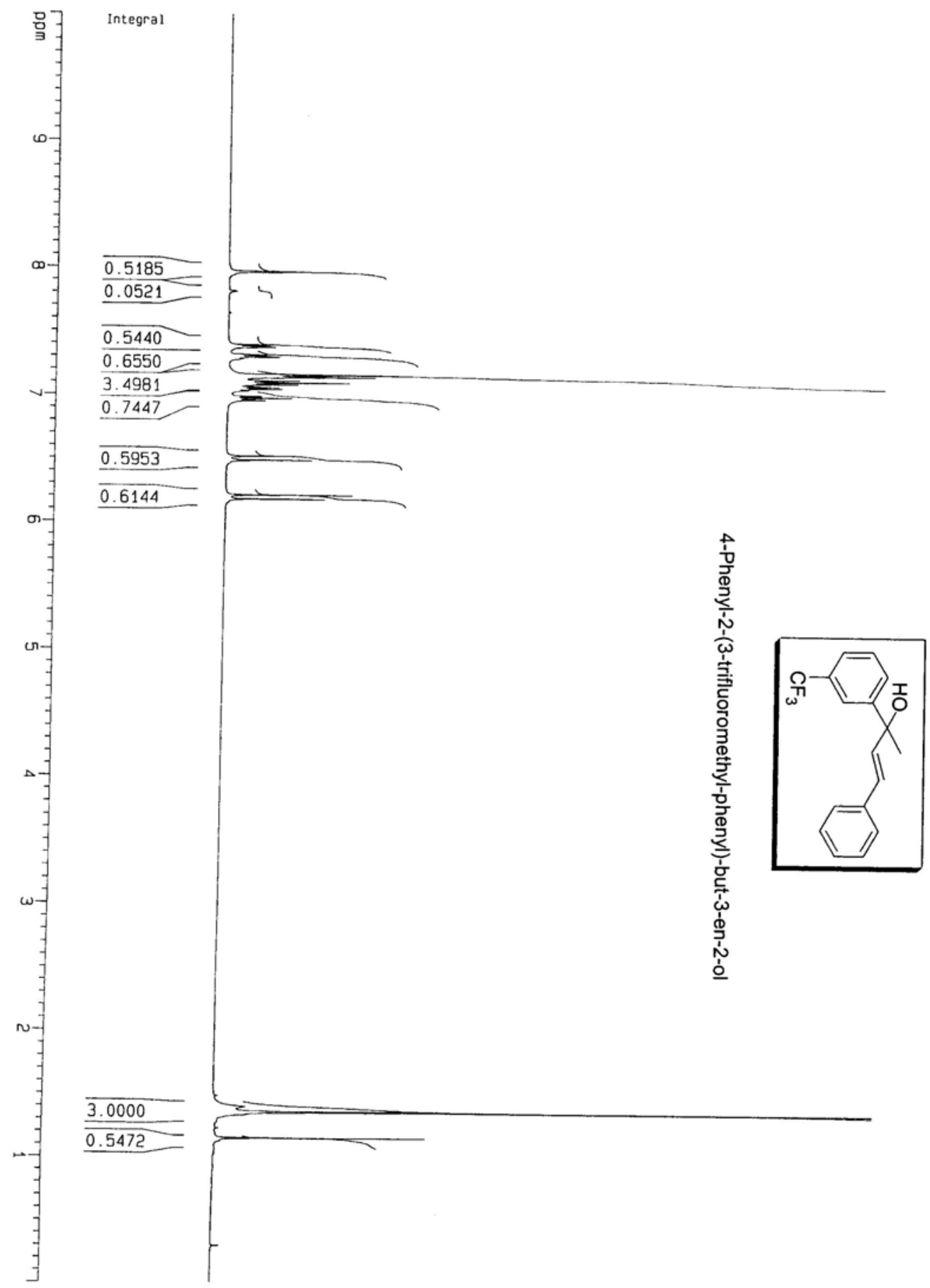




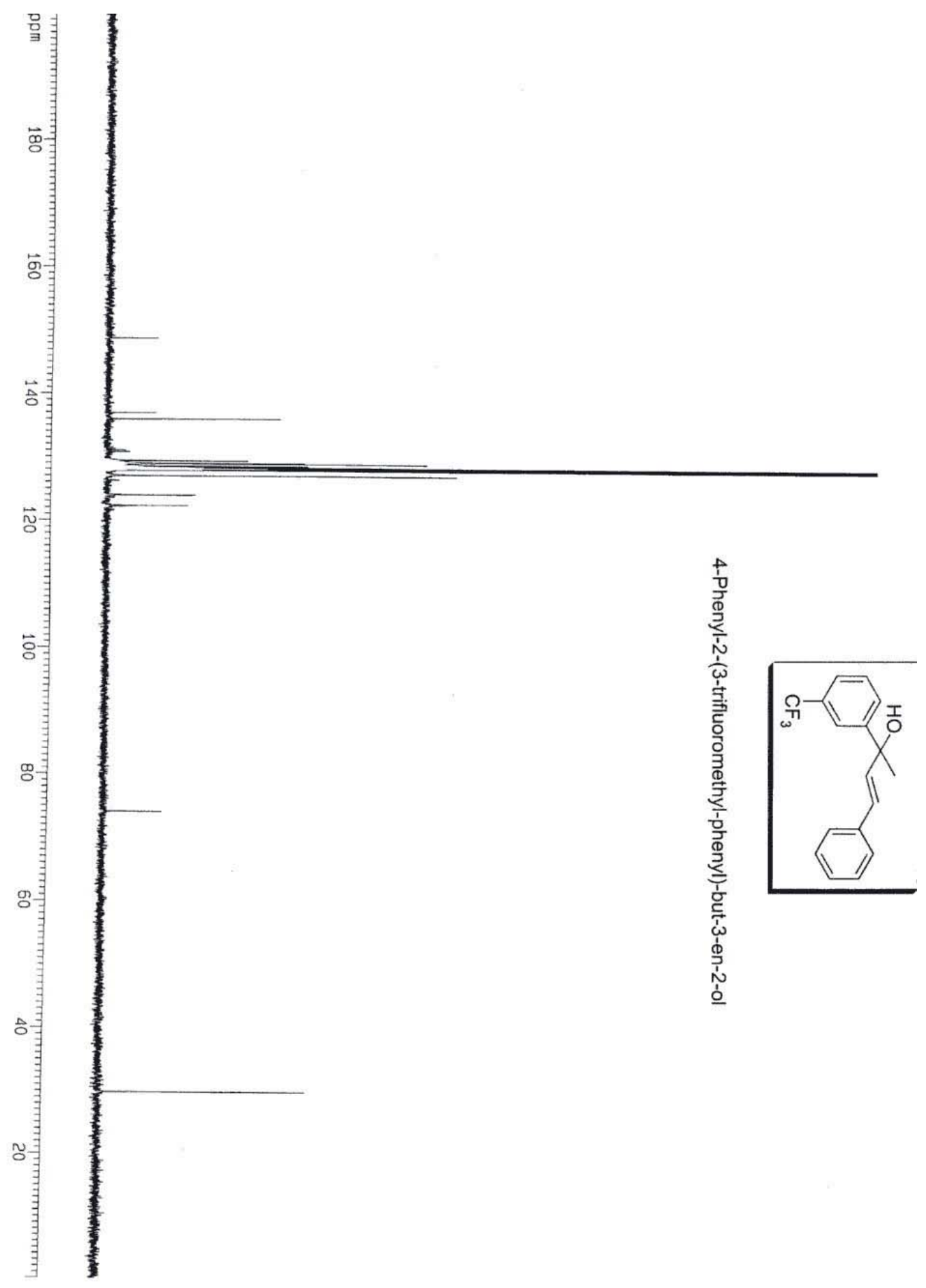




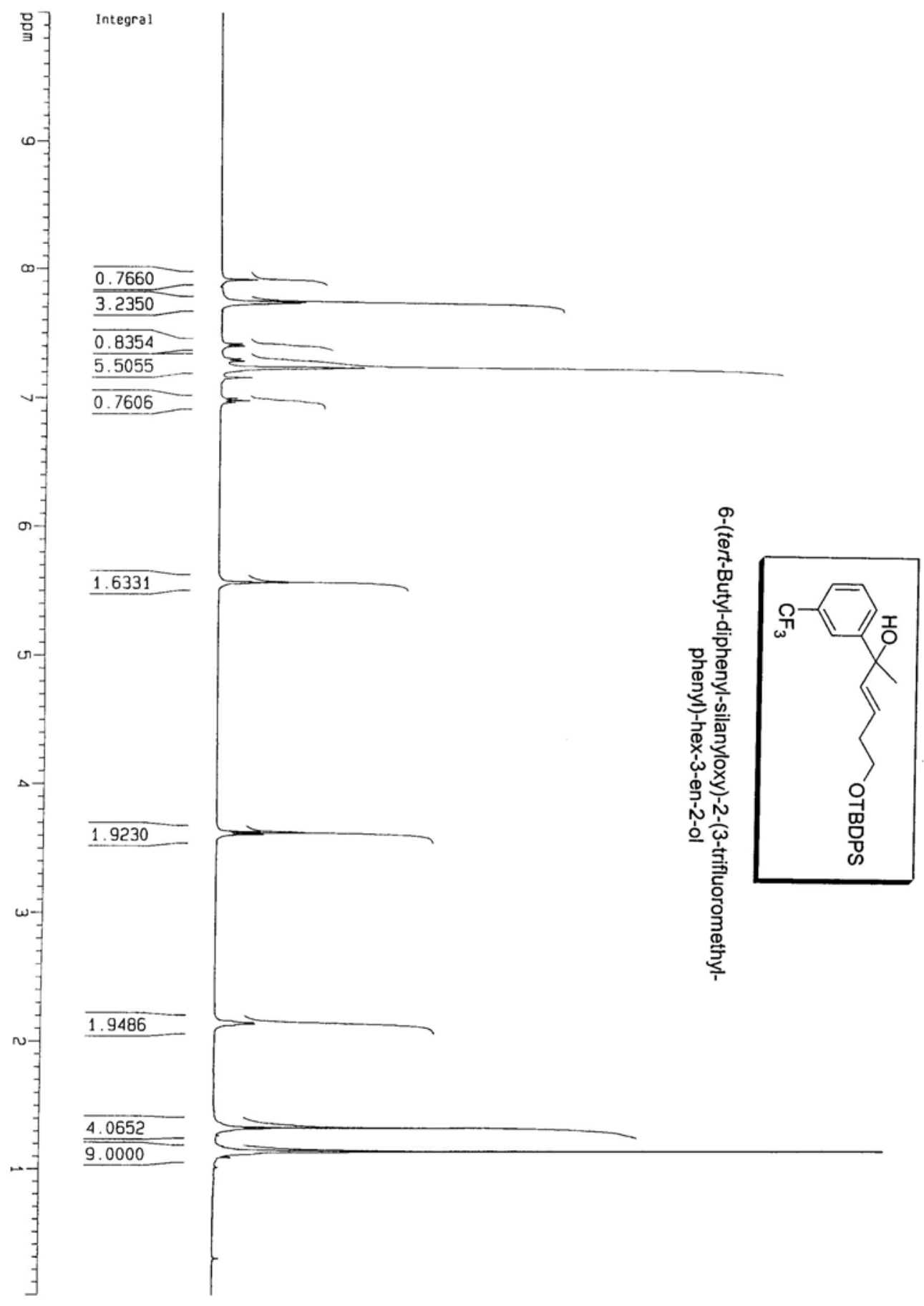



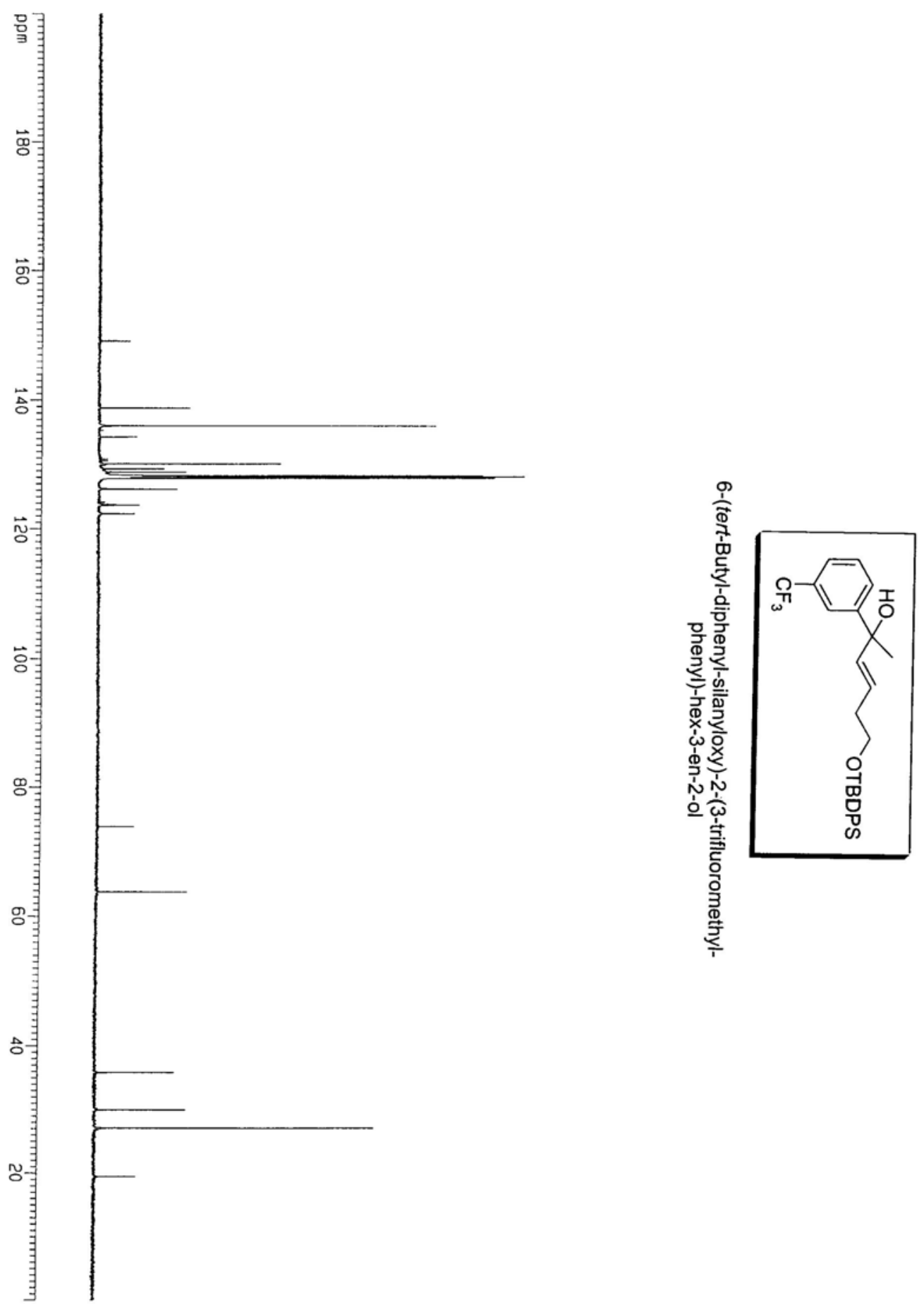


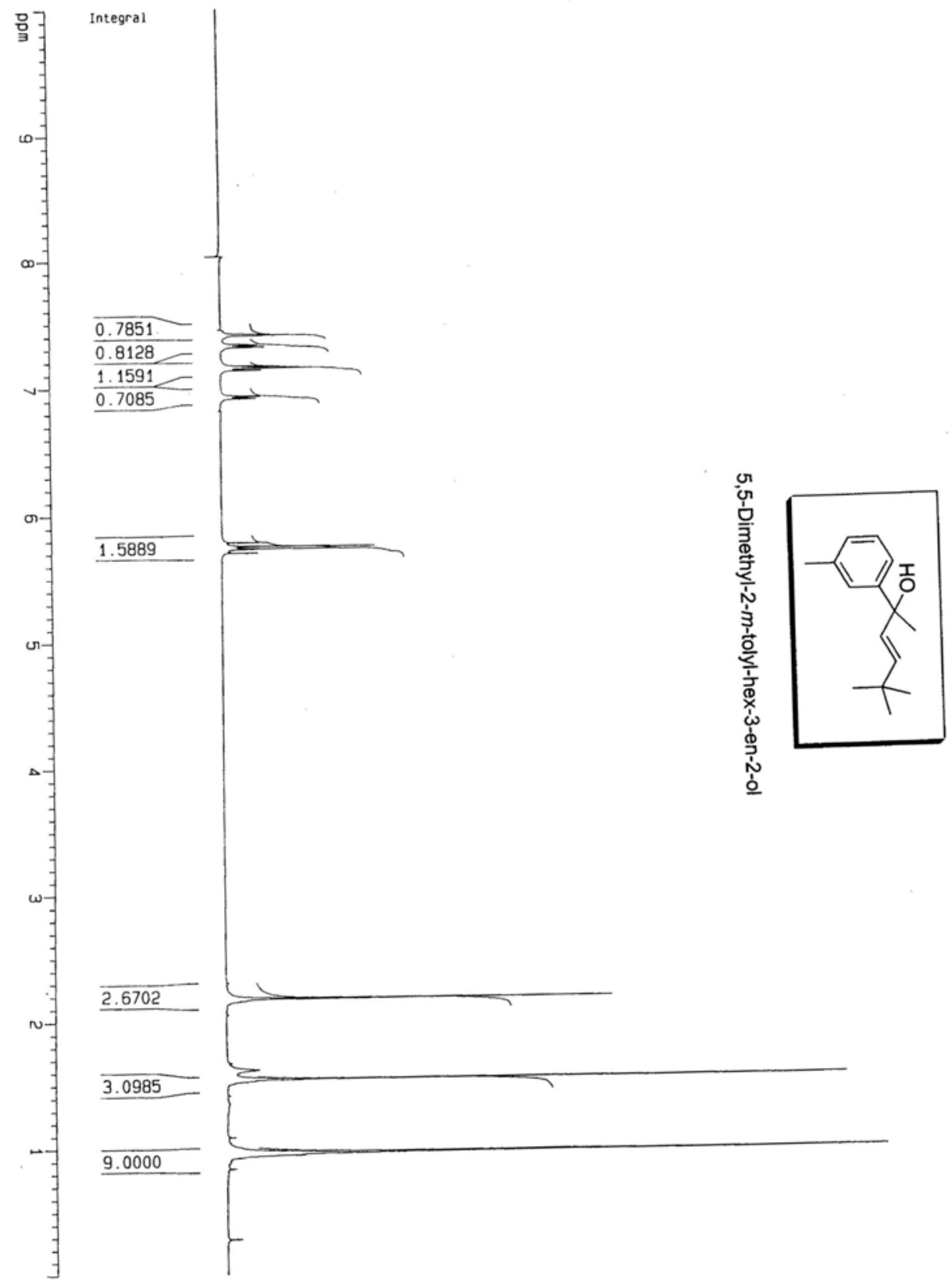



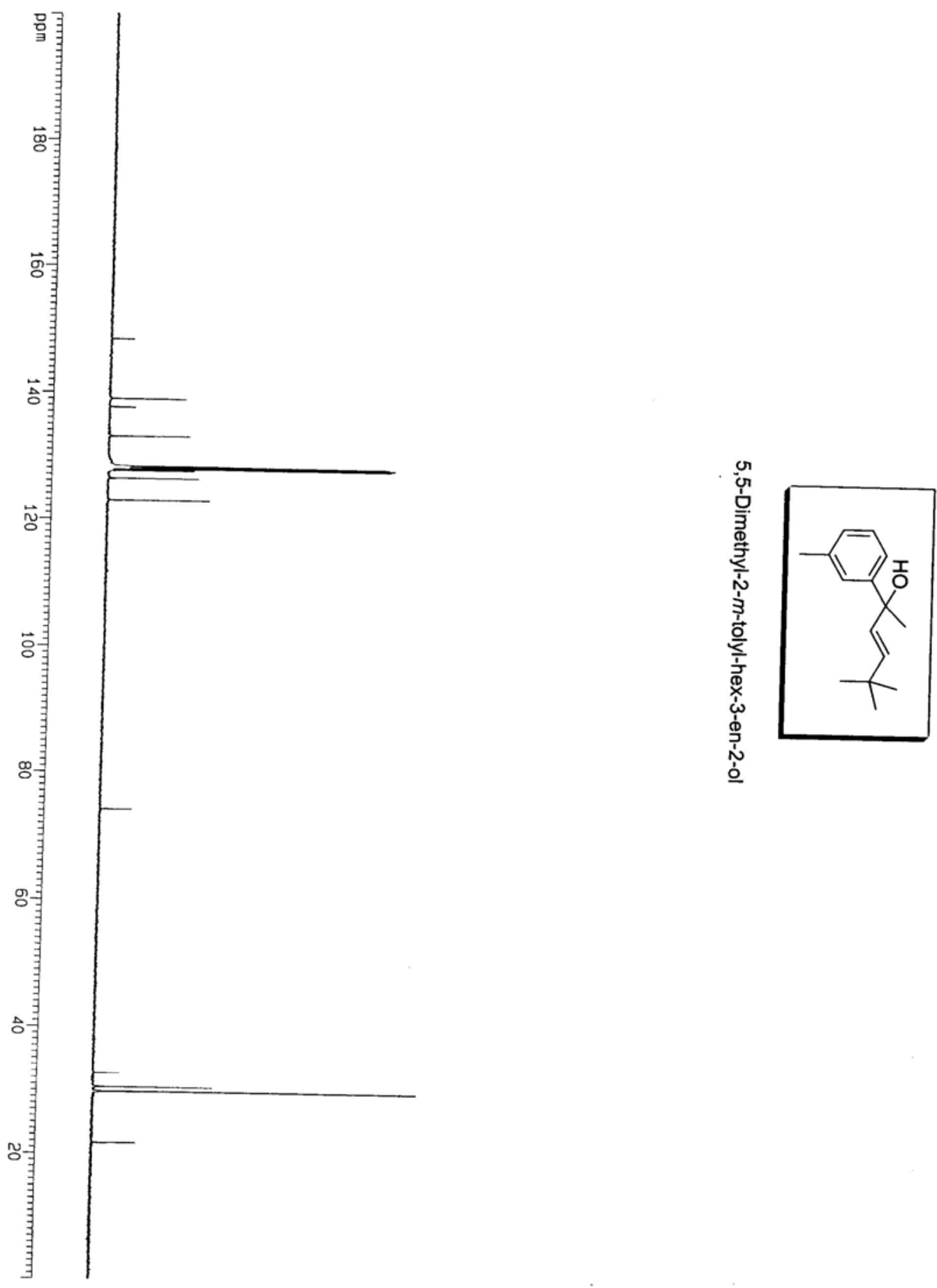


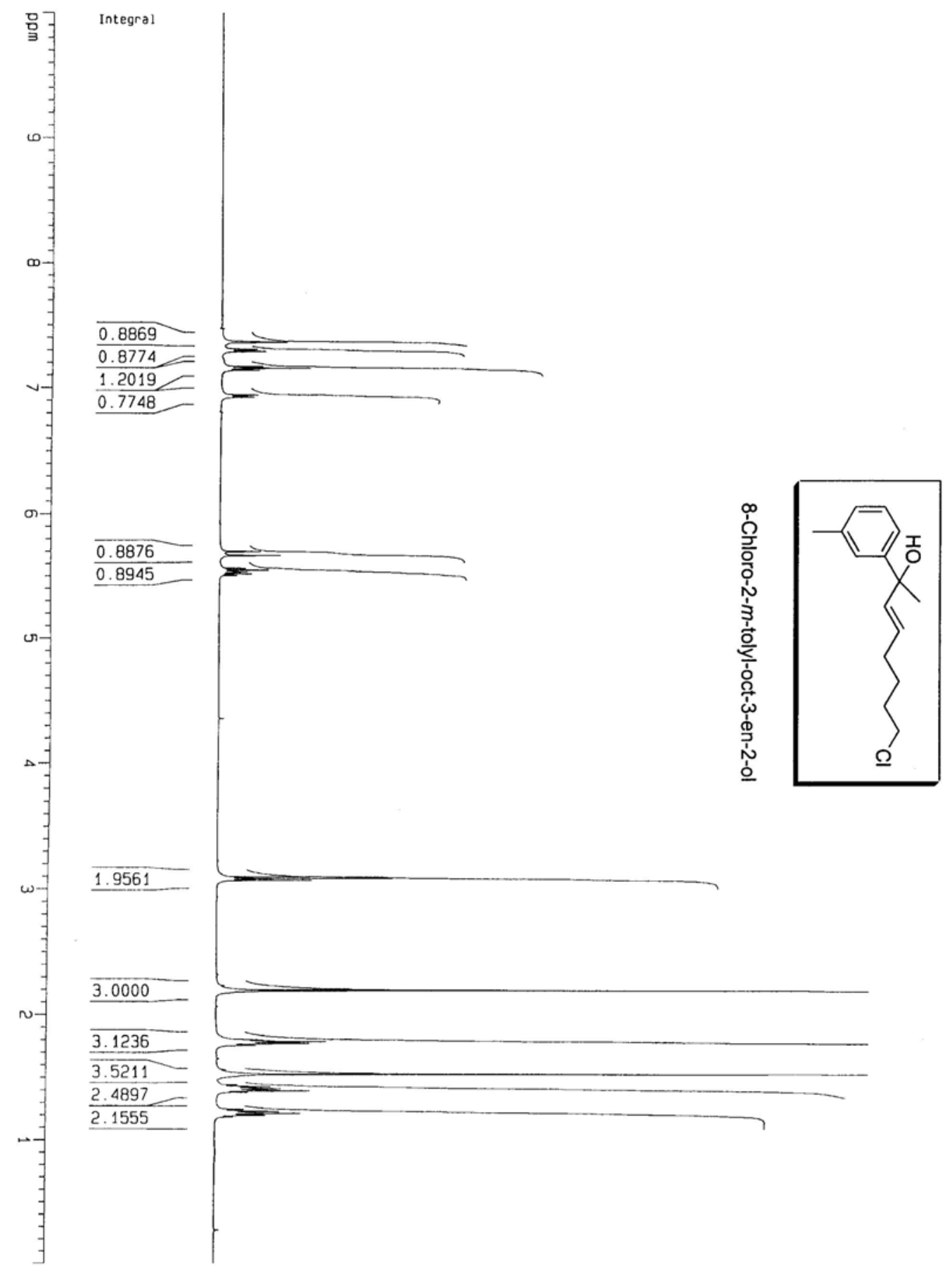



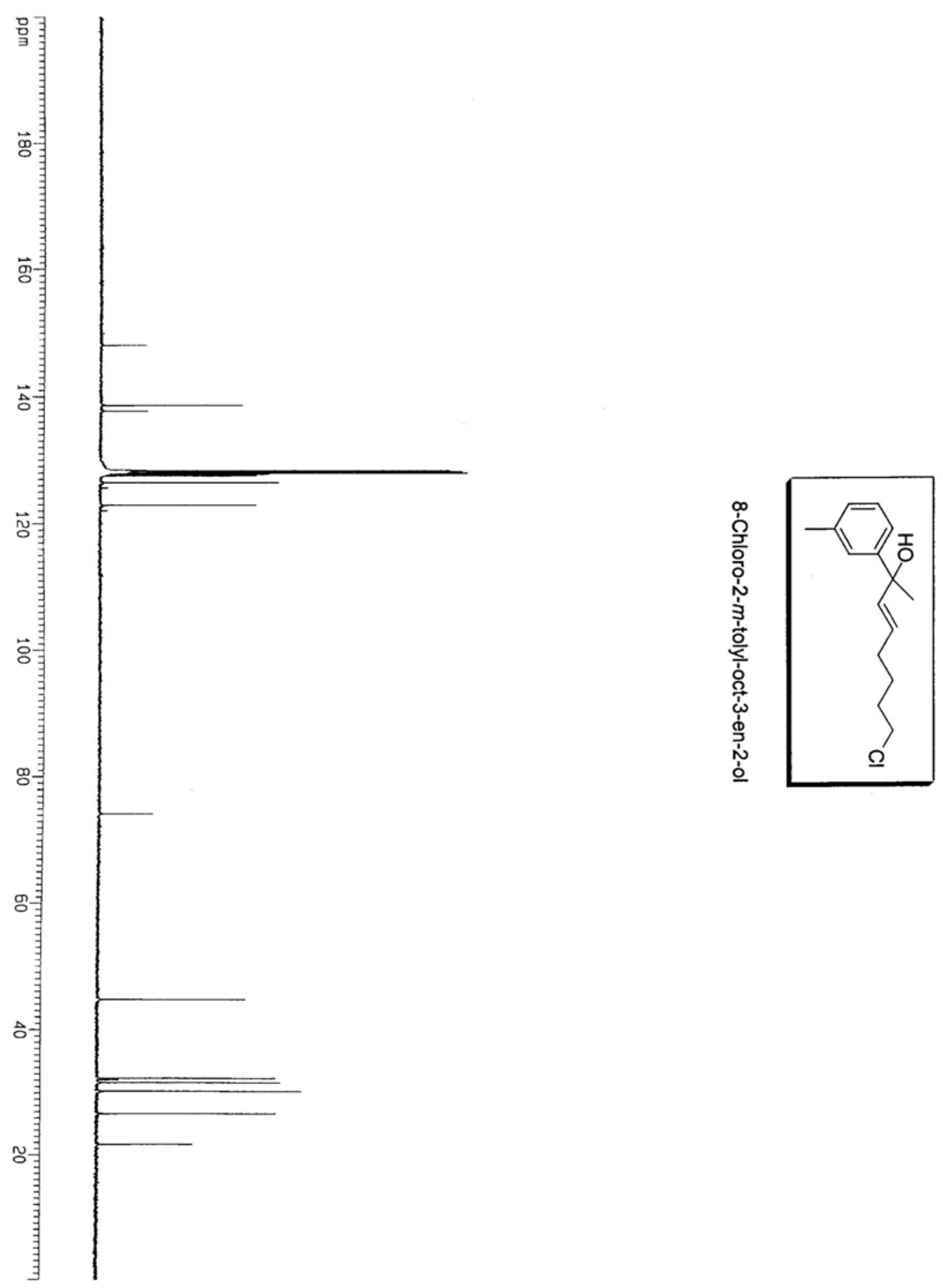


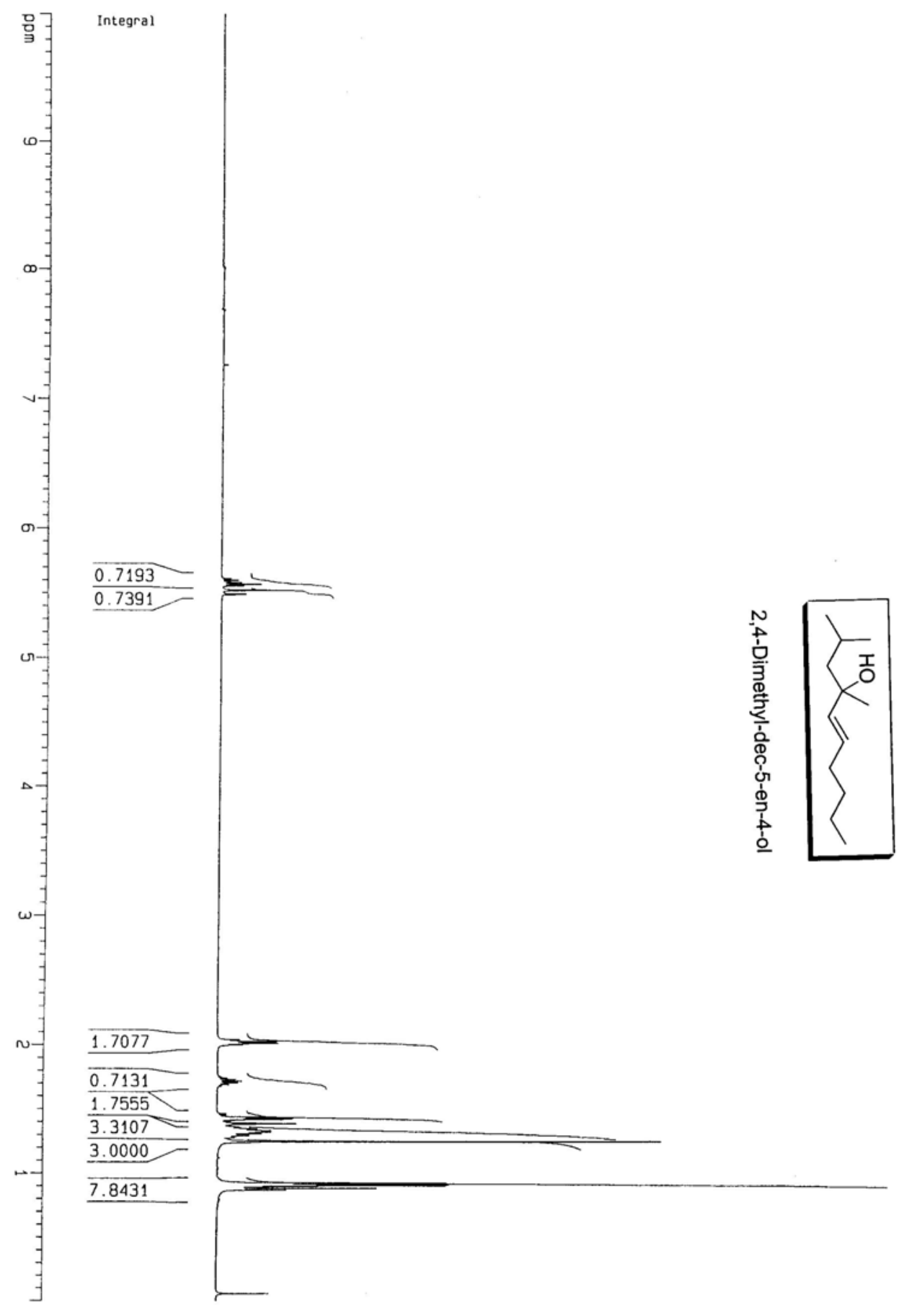



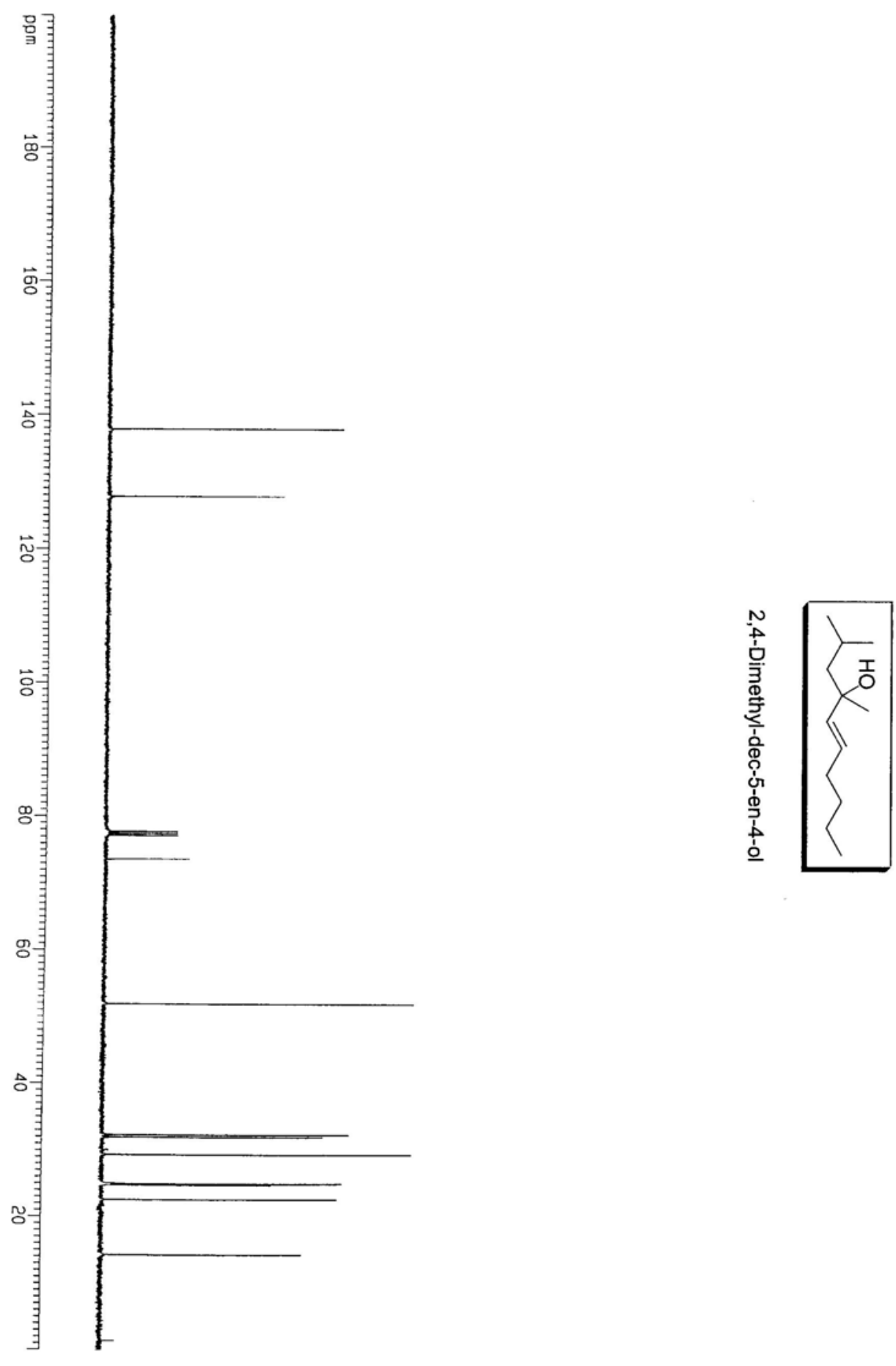


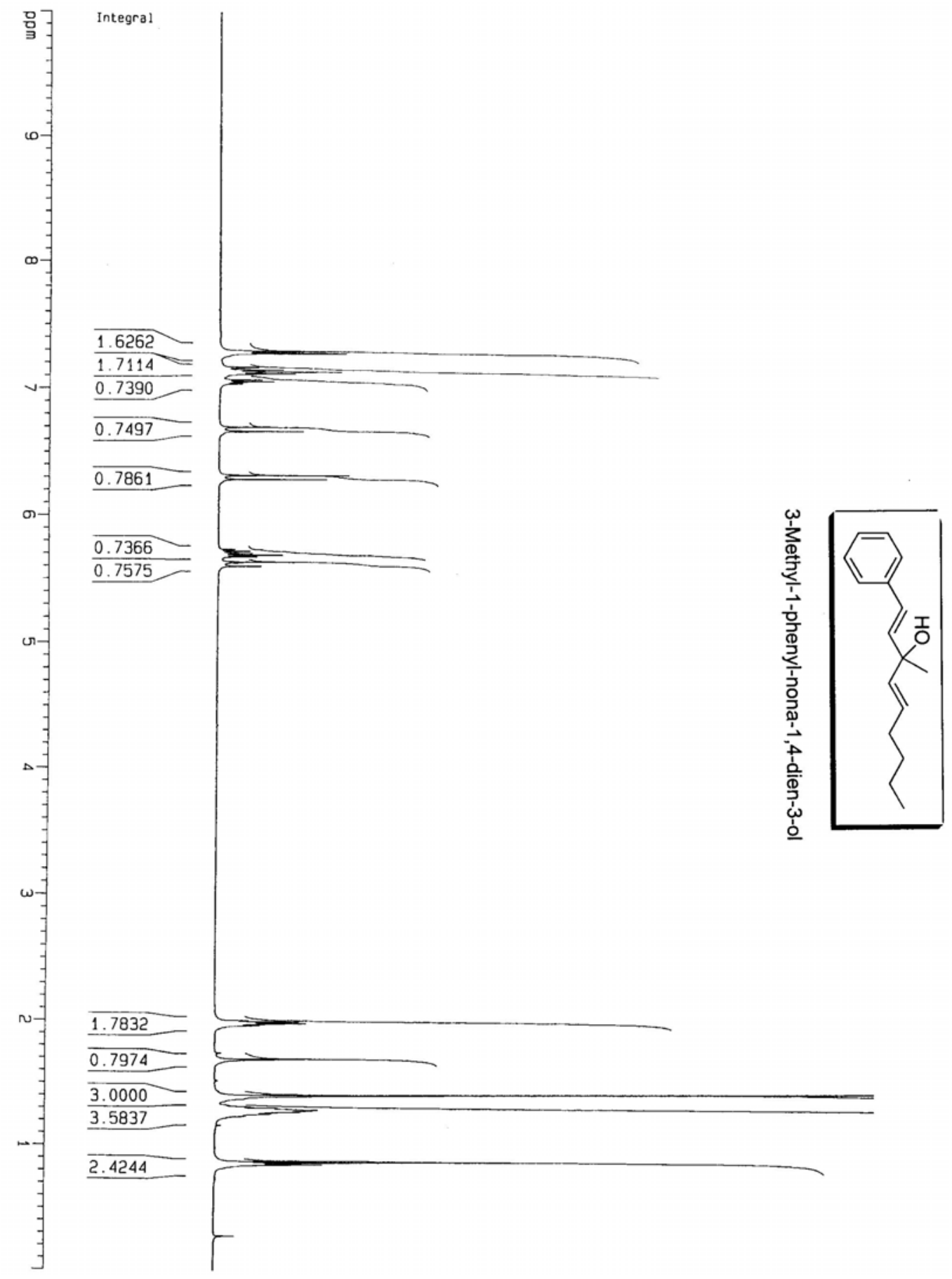



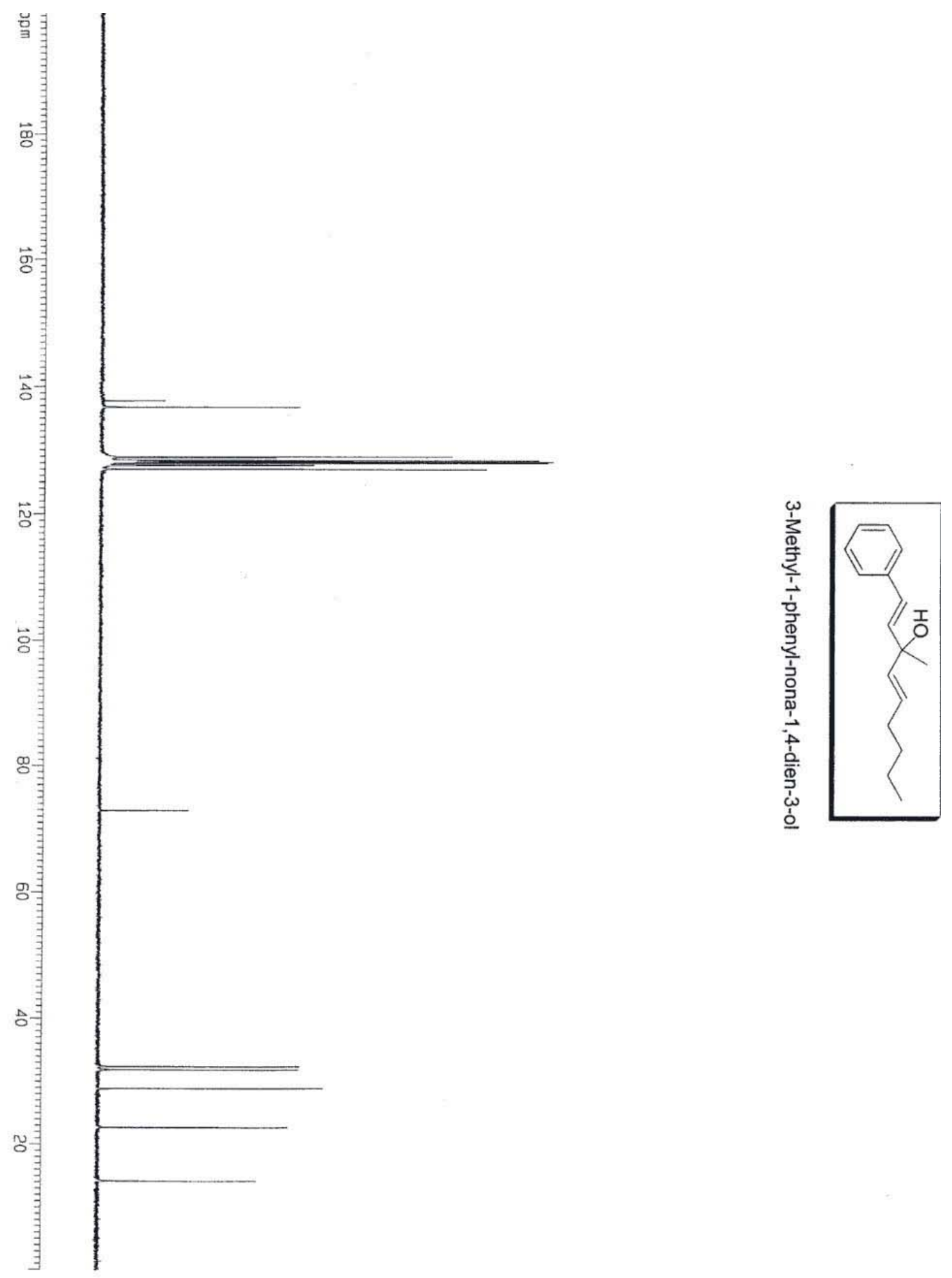


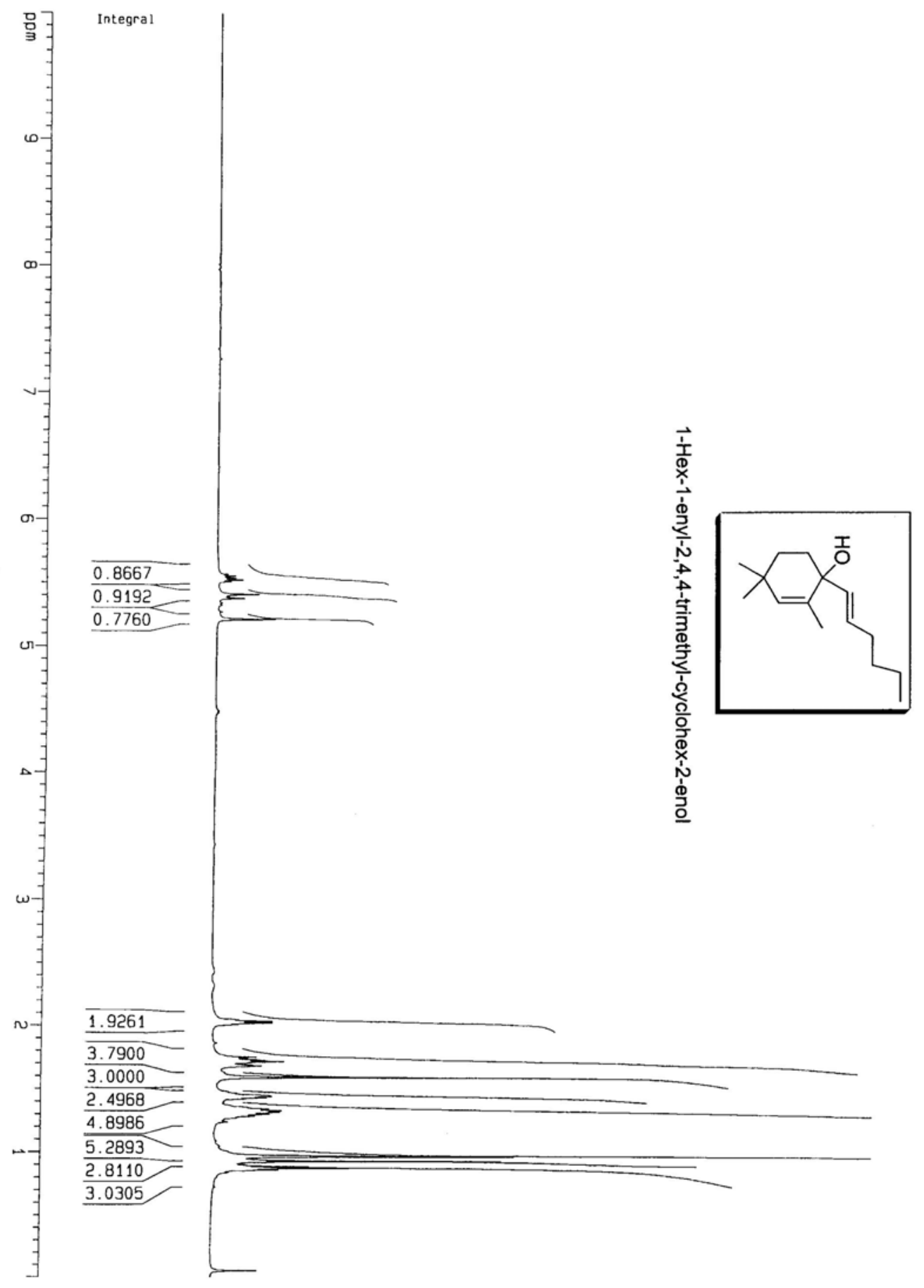



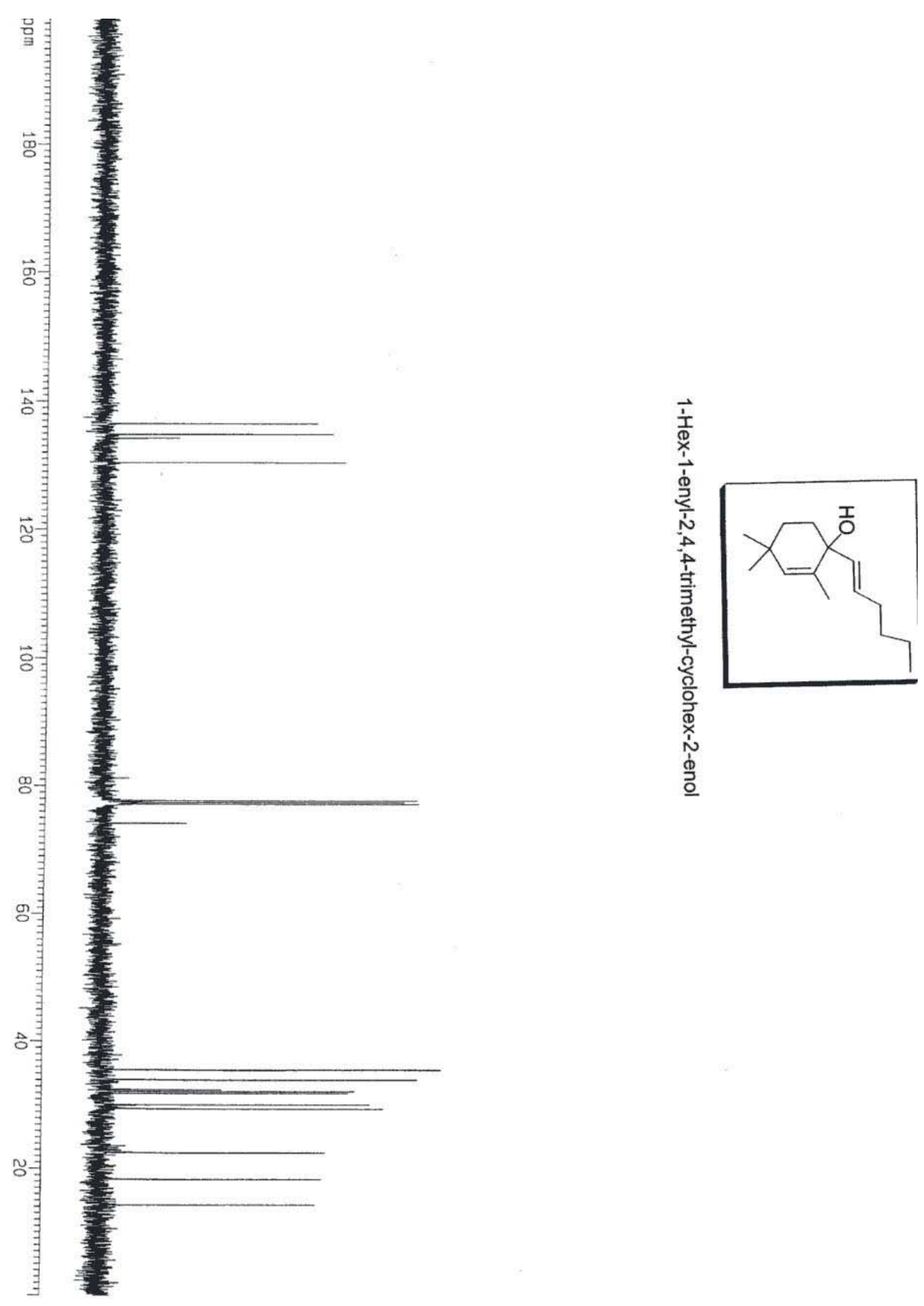


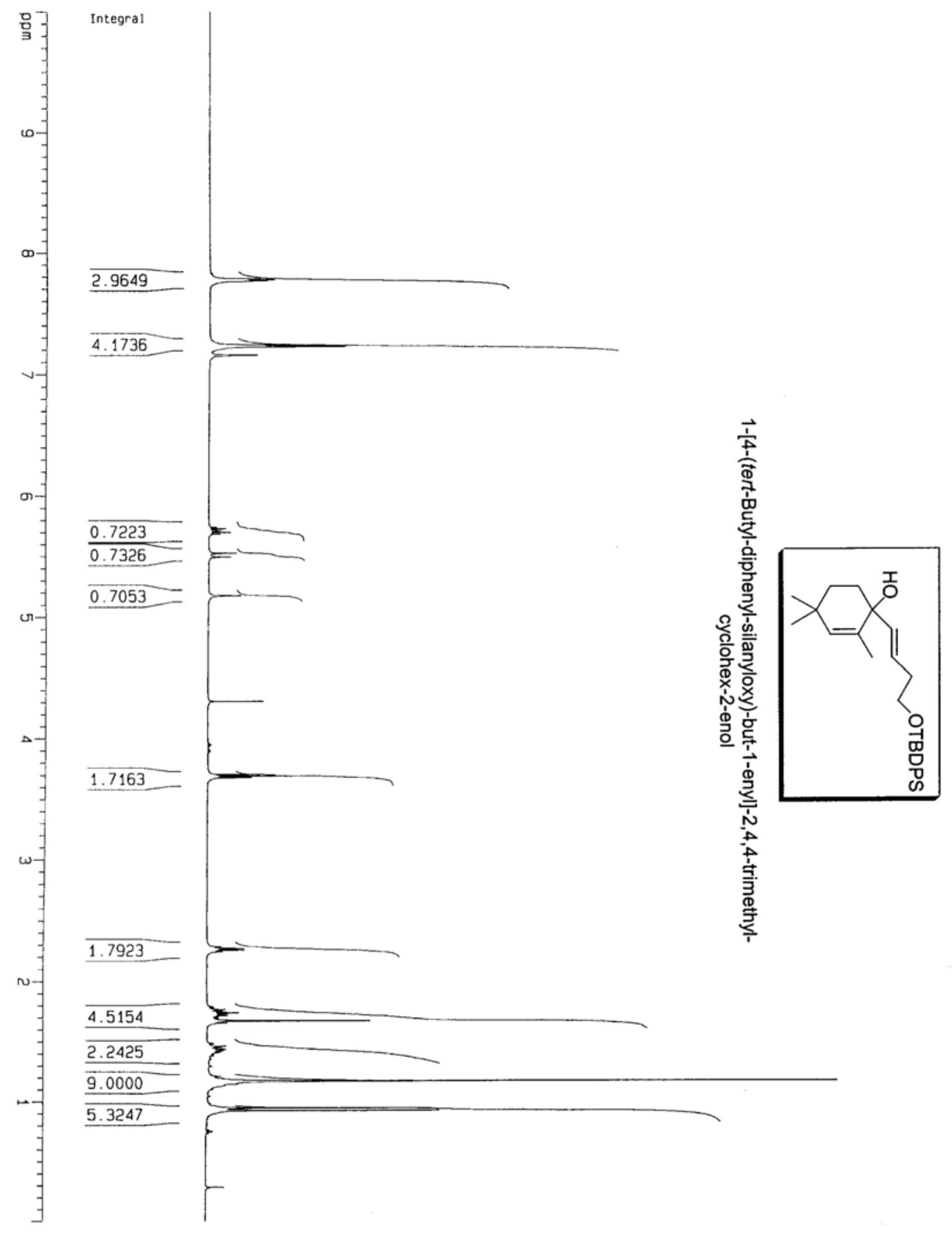




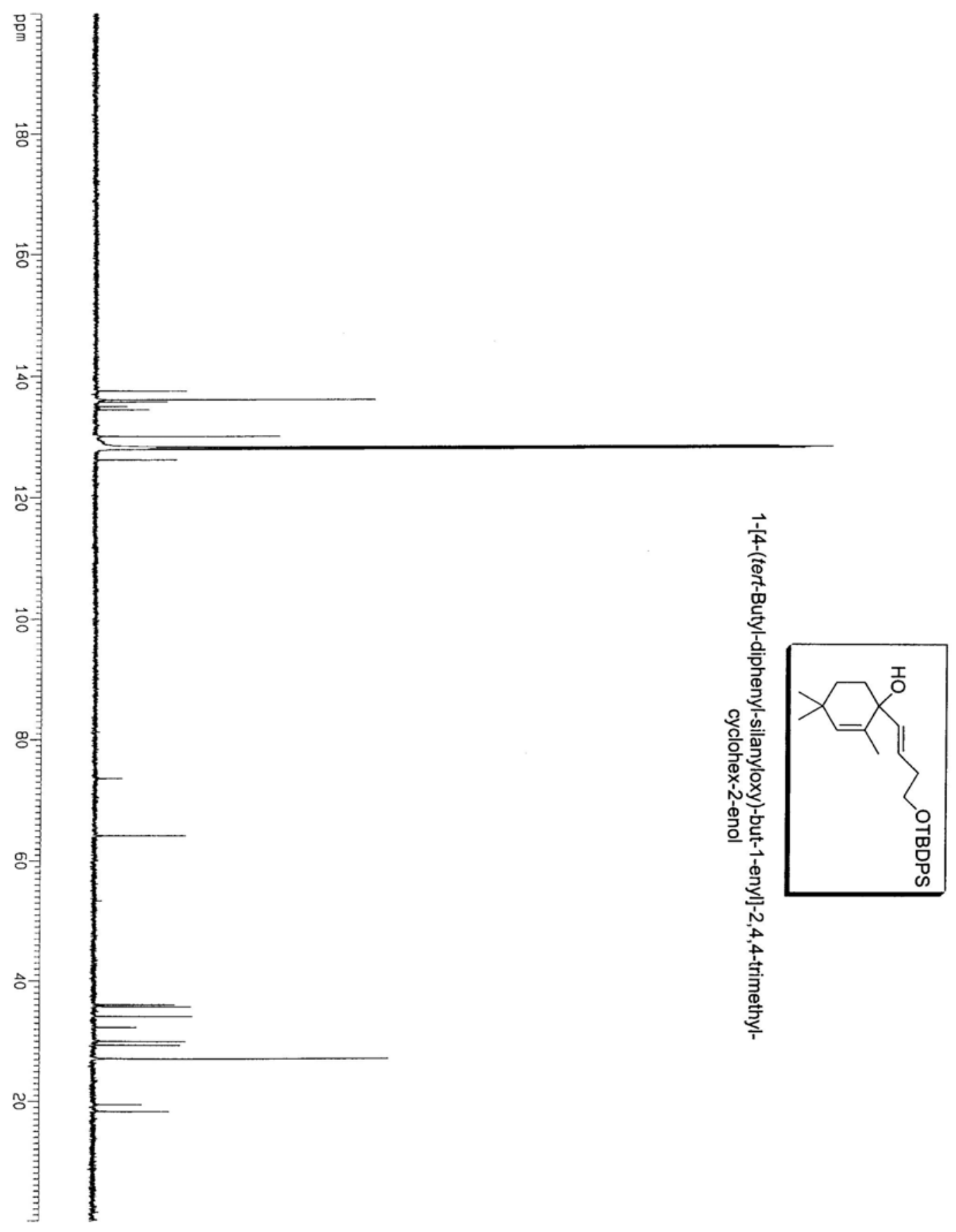




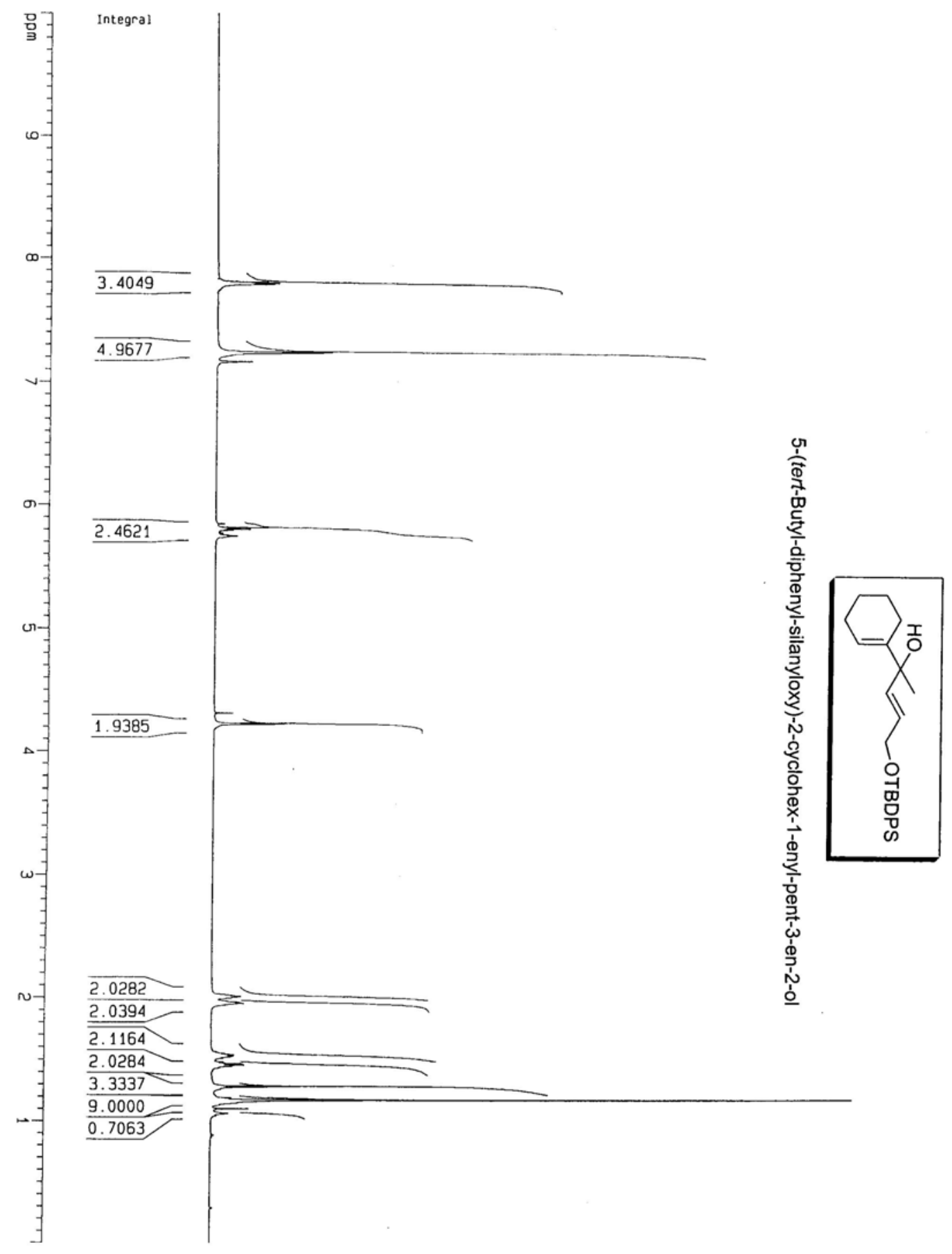




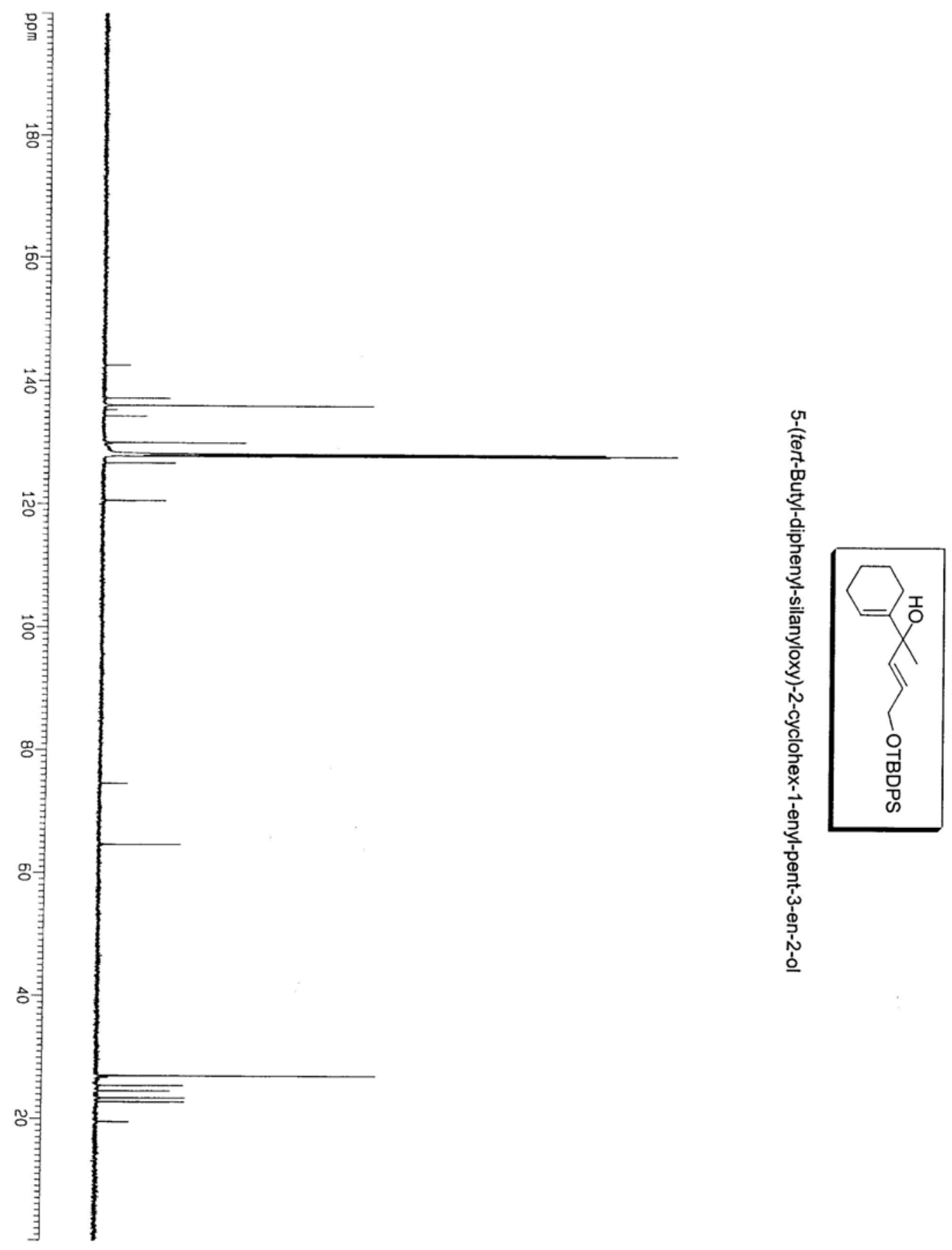




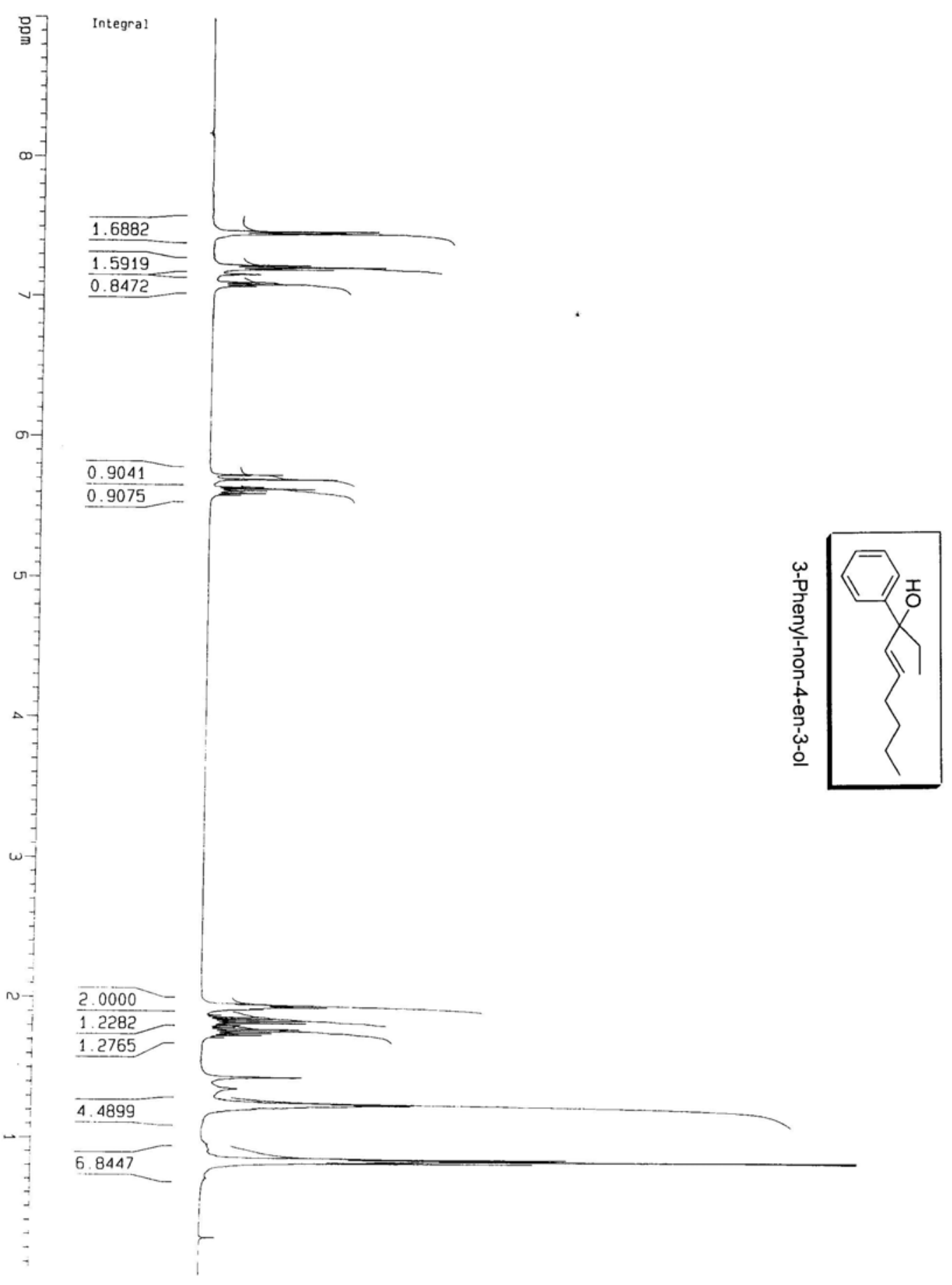




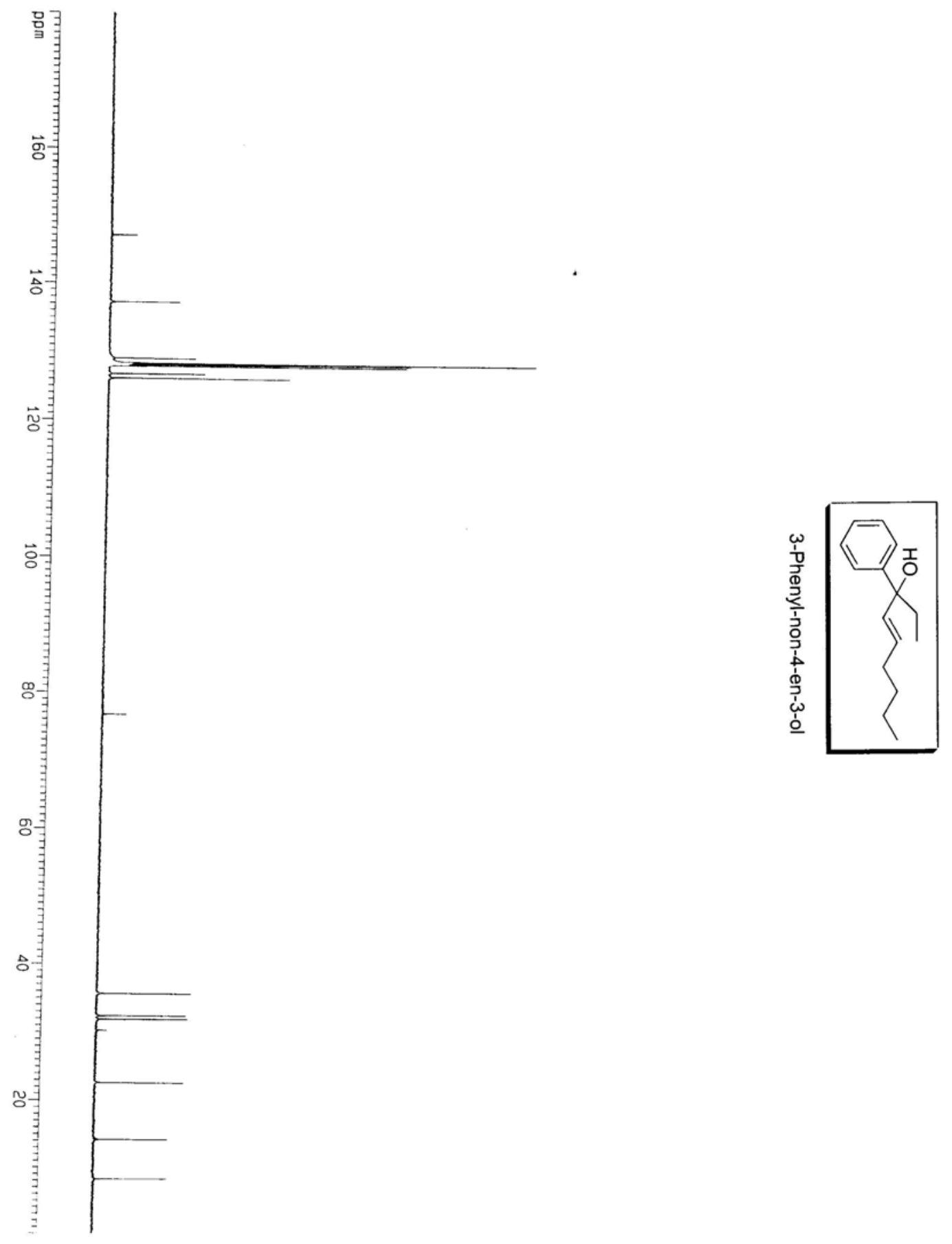




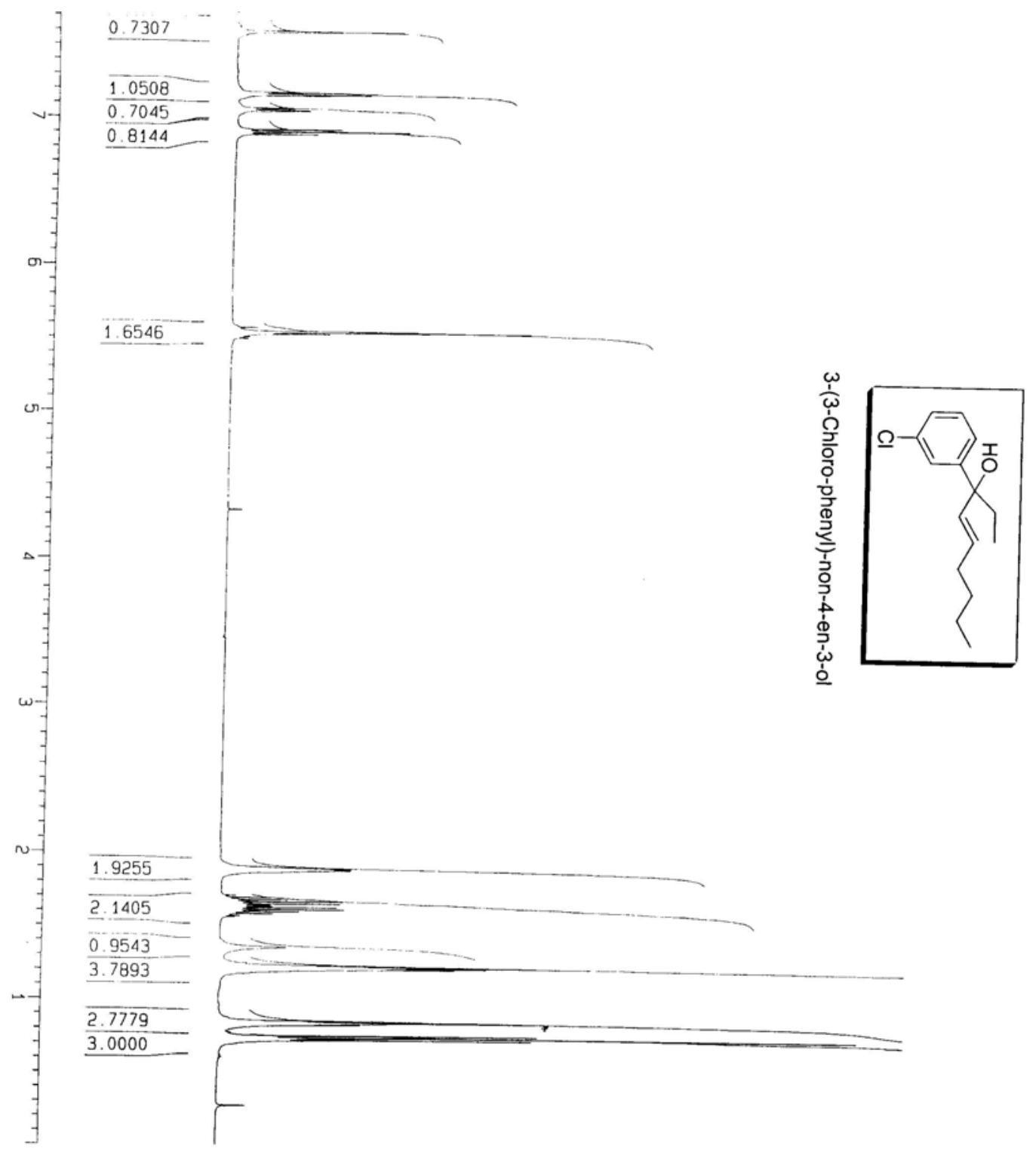



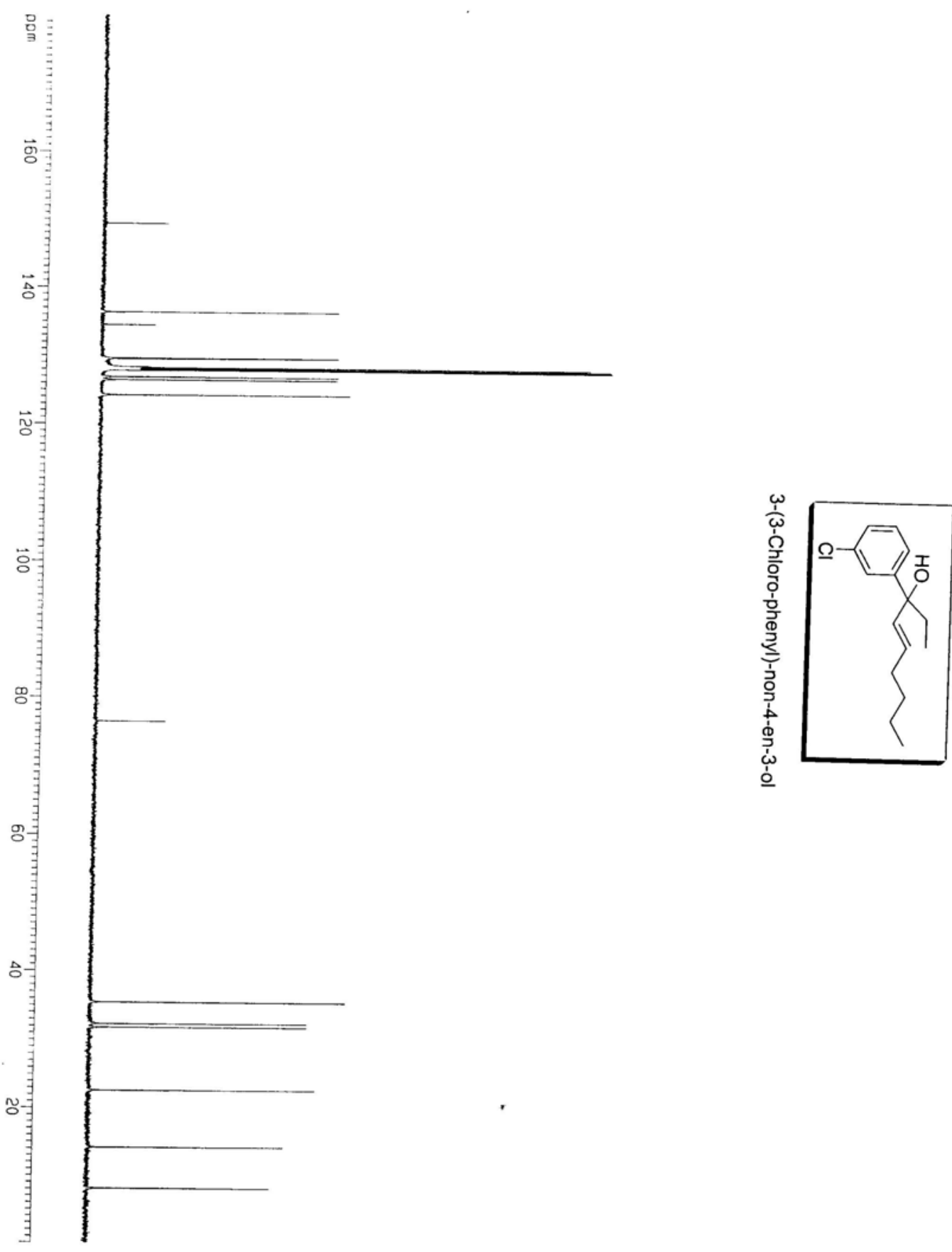